\title{
Characterizing the Dynamic Response of a Chassis Frame in a Heavy-Duty Dump Vehicle Based on an Improved Stochastic System Identification
}

\author{
Zhi Chen, ${ }^{1}$ Tie Wang, ${ }^{1}$ Fengshou Gu, ${ }^{1,2}$ and Ruiliang Zhang ${ }^{1}$ \\ ${ }^{1}$ Taiyuan University of Technology, Taiyuan, Shanxi 030024, China \\ ${ }^{2}$ University of Huddersfield, Queensgate, Huddersfield HD1 3DH, UK \\ Correspondence should be addressed to Tie Wang; wangtie57@163.com
}

Received 21 April 2015; Accepted 22 July 2015

Academic Editor: Carlo Trigona

Copyright (C) 2015 Zhi Chen et al. This is an open access article distributed under the Creative Commons Attribution License, which permits unrestricted use, distribution, and reproduction in any medium, provided the original work is properly cited.

\begin{abstract}
This paper presents an online method for the assessment of the dynamic performance of the chassis frame in a heavy-duty dump truck based on a novel stochastic subspace identification (SSI) method. It introduces the use of an average correlation signal as the input data to conventional SSI methods in order to reduce the noisy and nonstationary contents in the vibration signals from the frame, allowing accurate modal properties to be attained for realistically assessing the dynamic behaviour of the frame when the vehicle travels on both bumped and unpaved roads under different operating conditions. The modal results show that the modal properties obtained online are significantly different from the offline ones in that the identifiable modes are less because of the integration of different vehicle systems onto the frame. Moreover, the modal shapes between $7 \mathrm{~Hz}$ and $40 \mathrm{~Hz}$ clearly indicate the weak section of the structure where earlier fatigues and unsafe operations may occur due to the high relative changes in the modal shapes. In addition, the loaded operations show more modes which cause high deformation on the weak section. These results have verified the performance of the proposed SSI method and provide reliable references for optimizing the construction of the frame.
\end{abstract}

\section{Introduction}

The chassis frame in a heavy-duty truck is often subjected to extreme loads because the vehicle operates in different development areas, such as mining and construction sites where the road condition is commonly very poor and with high risks for driving. The frame is needed to be not only strong enough to withstand complicated loads but also sufficiently rigid to undertake high dynamic shocks, twists, and vibrations due to excitations of road irregularities. To meet these requirements, an accurate understanding of dynamic properties including modal frequency, damping, and shapes for the frame is necessary to minimize occurrences operating with structural resonances which may cause excessive dynamic stresses and result in structure failures, ride safety problem, ride discomfort, and high noise and vibration. In addition, accurate knowledge of these dynamic properties is also important to optimize the structures for a lightweight frame which are being paid more attention in recent years for improving fuel efficiency and reducing greenhouse effects.

However, it has been found that most of studies employ a combination of finite element (FE) analysis and an experimental verification [1-3] to determine the dynamic properties. During the experimental verification, the free frame without the integration of other components is excited by controlled or known inputs such as impulsive forces by an impact hammer. Then, both the input excitation and output responses are measured to estimate the modal parameters. The results obtained offline can be an important reference for predicting the dynamics of overall system in a vehicle design process. However, in reality, the frame is assembled with different subsystems and can behave very differently because of the effect of added distribution masses and different nonstandard constraints which are difficult to be modelled in FE calculation and are measured through conventional controlled excitations. Therefore, an online identification 


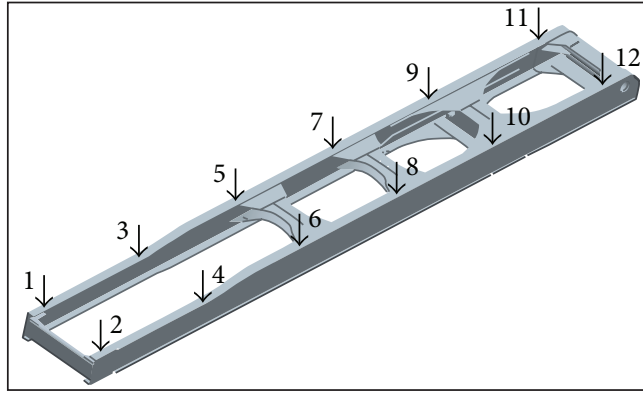

(a)

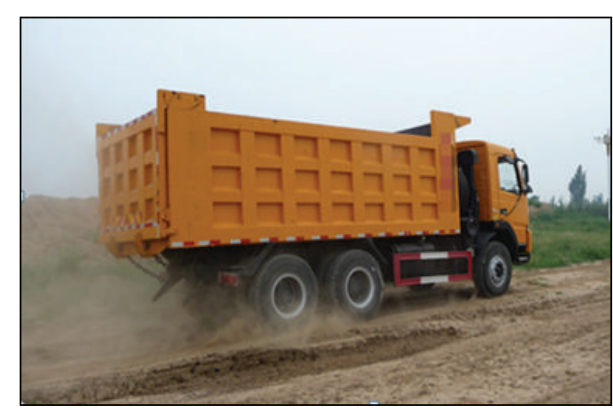

(b)

FIgURE 1: Testing structures and system. (a) The construction of the frame and (b) the dump truck on road.

method is more appreciated in order to obtain the real dynamic behaviours when the frame is applied by different dynamic loads when the vehicle operates close to its real applications. The results obtained in such way can then provide reliable support for assessing and refining the overall dynamic performance of a vehicle.

Amongst the many different techniques of obtaining the dynamic properties, the operational modal analysis (OMA) has the most potential for online applications because it needs only output data which is easier to be obtained. Therefore, it has been used widely for the analysis of different civil structures [4-6]. Furthermore, many variants of OMA can be potentially useful for online dynamics analysis of the frame $[7,8]$. In comparative studies, the reference-based stochastic subspace identification (SSI/ref) [7] method was deemed to be a more accurate, robust, and efficient identification for OMA [9] and has been intensively explored recently in the field of ambient vibration data based modal identification. Moreover, by considering the road excitations as random inputs, subspace identification methods were investigated to estimate the vehicle handling dynamic model and predict the vehicle handling performances $[10,11]$ using data from road tests. Therefore, this study is also based on this approach to implement the online analysis of frame dynamic responses.

However, as the road excitations are not completely stationary and the response data can be heavily influenced by different noises such as secondary vibrations from the components connected to the frame and interfering excitations from power trains, it has been found that the direct use of measured signals, including their covariances as the input for the SSI/ref algorithm, can lead to numerous deceptive modes and it is difficult to obtain a consistent result for the frame dynamics analysis. This means that a noise suppression method is required to preprocess the measured signals to improve the signal to noise ratio (SNR) significantly for effective use of SSI/ref methods. On the other hand, the so-called Natural Excitation Technique (NExT) uses the correlation technique for modal identification. It was shown that the cross-correlation signals between two responses to white noise inputs are of the same form as free-vibration decay or impulse responses. In studies of $[12,13]$, the use of cross-correlation functions between response channels was proposed and showed effectiveness for both stationary and nonstationary white noise ambient excitation signals for modal parameters identification. Based on these studies including the super performance of correlation function in extracting periodic signals in strong noisy data, an average correlation signal based SSI/ref is therefore proposed to suppress the noise and nonstationary responses measured on the frame for identifying its dynamic properties.

The rest of the paper has four further sections. Section 2 outlines the theoretical background in developing the average correlation signal based SSI. Section 3 verifies the performance of the method by using an offline test. Section 4 presents the results and discussion for the modal parameters obtained through online identification. Finally, the conclusions are given in Section 5.

\section{Background and Methodology}

2.1. Frame Vibration Responses. As illustrated in Figure 1, the frame concerned in this study is used in a 25-ton heavyduty dump truck. Its structure has been newly optimized for increased strength, handling stability, and lightweight performance. By using offline FE analysis and hammer based modal test evaluation, the new frame was confirmed to have a frequency increase of about $5 \mathrm{~Hz}$ for the first 10 modes below $100 \mathrm{~Hz}$ but its weight was $4.3 \%$ less than the original one.

To validate these improvements in real operations, this study develops an online modal analysis method to assess the dynamic characteristics of the frame through vibration measurements. In particular, it is to check and evaluate if any significant vibration modes occur when the vehicle operates on different unpaved roads under loaded and unloaded conditions. However, as the vehicles often operate on temporary unpaved roads which have very oscillatory surfaces with many large valleys and sharp summits, it means that the frame can be subjected to very high dynamic loads due to these primary excitations. In addition, its power train, cab, joints, and containers also cause significant secondary excitations which make measured signals very unclean. As shown in Figure 2, the vibration signals, which were measured with twelve acceleration sensors uniformly distributed on the frame, illustrated in Figure 1 when the vehicle travelled on unpaved roads at about $20 \mathrm{~km}$ with load condition, show that the signals are very nonstationary with many local large 

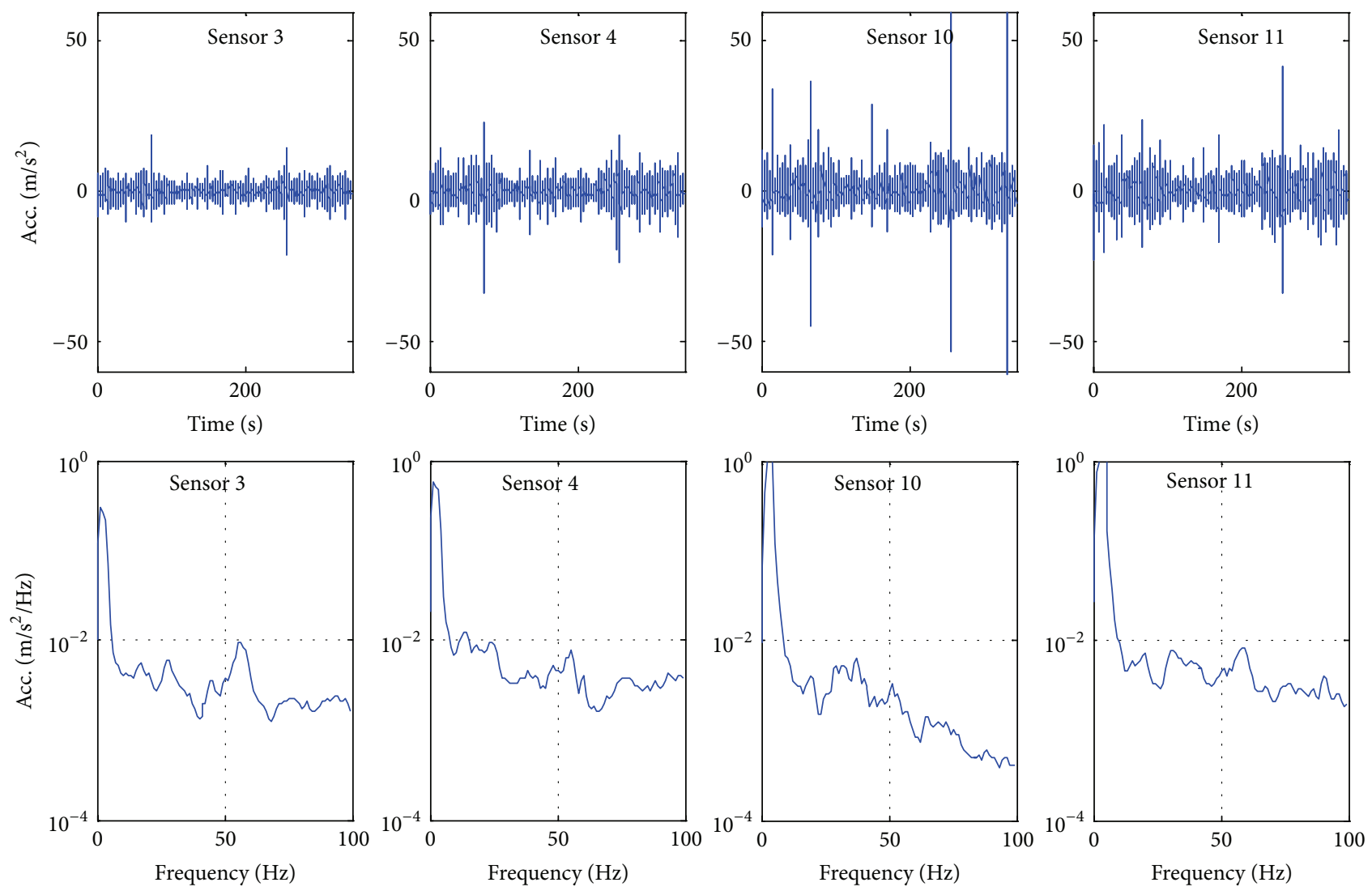

Figure 2: Raw vibration signals and their spectra.

responses. Their corresponding spectra show that the signal energy is mainly below $10 \mathrm{~Hz}$ due to the responses of suspension system effects. However, the frequency range from $10 \mathrm{~Hz}$ to $100 \mathrm{~Hz}$, which is of interest for the frame dynamic analysis, shows much lower amplitudes and wider spread patterns. These show that the vibration contents contain high levels of noise and interfering components. Therefore, it is difficult to perform modal analysis using conventional OMA methods.

\subsection{Reference-Based Stochastic Subspace Identification} (SSI/ref). The stochastic subspace identification is developed from the state-space equations. For a vibration system with $n$ degrees of freedom (DOF) system with $l$ measurements, they are expressed as

$$
\begin{aligned}
x(k+1) & =\mathbf{A} x(k)+\mathbf{w}(k) \\
y(k) & =\mathbf{C} x(k)+\mathbf{v}(k),
\end{aligned}
$$

where the system matrix is $\mathbf{A}=e^{\mathbf{A}_{\mathrm{c}} \Delta t}$, the output allocation matrix is $\mathbf{C} \in R^{l \times 2 n}, \mathbf{w} \in R^{n \times 1}$ is process noise vector, $\mathbf{v} \in R^{l \times 1}$ is the measurement noise vector to the output equation with output vector $y \in R^{l \times 1}$, and state variable vector is

$$
\dot{x}(t)=\left(\begin{array}{cc}
\mathbf{0}_{n \times n} & \mathbf{I}_{n \times n} \\
-\mathbf{M C}_{\mathbf{v}} & -\mathbf{M K}
\end{array}\right) x(t)=\mathbf{A}_{\mathbf{c}} x(t)
$$

which is derived from the vibration system

$$
\mathbf{M} \ddot{u}(t)+\mathbf{C}_{\mathbf{v}} \dot{u}(t)+\mathbf{K} u(t)=\mathbf{F}(t),
$$

where $\mathbf{M}, \mathbf{C}_{\mathbf{v}}$, and $\mathbf{K} \in R^{n \times n}$ denote the mass, stiffness, and damping matrices, respectively, $\ddot{u}(t), \dot{u}(t)$, and $u(t) \in R^{n \times 1}$ are the acceleration vector, the velocity vector, and displacement vector at continuous time $t$, and $\mathbf{F}(t) \in R^{n \times 1}$ is the exciting force vector.

With state-space representation, modal parameters of frequency $f_{i}$, damping ratio $\xi_{i}$, and vectors $\phi$ can be determined using matrices $\mathbf{A}$ and $\mathbf{C}$ through

$$
\begin{aligned}
f_{i} & =\frac{\lambda_{i}^{c}}{2 \pi}, \\
\xi_{i} & =\frac{\operatorname{Re}\left(\lambda_{i}^{c}\right)}{\left|\lambda_{i}^{c}\right|}, \\
\phi & =\mathbf{C} \Phi,
\end{aligned}
$$

where the continuous-time system its eigenvalues is from its discrete one by $\lambda_{i}^{c}=\ln \left(\lambda_{i}\right) / \Delta t$ which is the diagonal value of $\boldsymbol{\Lambda}$ obtained by the decomposition of $\mathbf{A}=[\boldsymbol{\Phi}] \boldsymbol{\Lambda}[\boldsymbol{\Phi}]^{-1}$. $[\boldsymbol{\Phi}]$ contains eigenvectors as columns.

In implementing the stochastic subspace based system identification for estimating $\mathbf{A}$ and $\mathbf{C}$, output data $y$ with a length $j$ (assumed to be infinitive) from $l$ sensors are 
organized into a Hankel matrix with $2 i$ block rows and $j$ columns according to the reference-based SSI scheme in [7]:

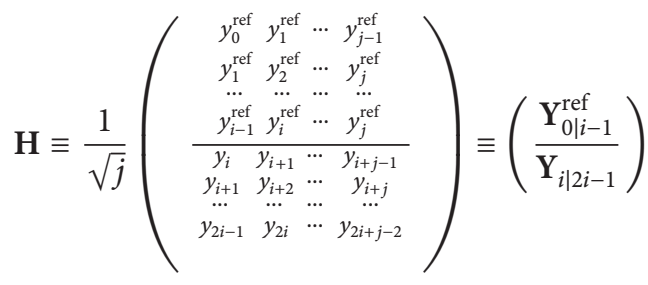

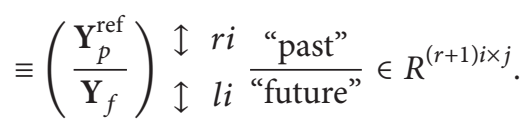

Using the past reference submatrix $\mathbf{Y}_{p}^{\mathrm{ref}}$ from $r(r \leq l)$ channels and future submatrix $\mathbf{Y}_{f}$, a covariance matrix between all outputs and the reference channels can be calculated and gathered in a block Toeplitz matrix as

$$
\begin{aligned}
\mathbf{T}_{1 \mid i}^{\mathrm{ref}} & =\mathbf{Y}_{f} \mathbf{Y}_{p}^{\mathrm{ref}^{T}}=\left(\begin{array}{cccc}
\Lambda_{i}^{\mathrm{ref}} & \Lambda_{i-1}^{\mathrm{ref}} & \cdots & \Lambda_{1}^{\mathrm{ref}} \\
\Lambda_{i+1}^{\mathrm{ref}} & \Lambda_{i+2}^{\mathrm{ref}} & \cdots & \Lambda_{2}^{\mathrm{ref}} \\
\cdots & \cdots & \cdots & \cdots \\
\Lambda_{2 i-1}^{\mathrm{ref}} & \Lambda_{2 i-2}^{\mathrm{ref}} & \cdots & \Lambda_{i}^{\mathrm{ref}}
\end{array}\right) \\
& \in R^{l i \times r i} .
\end{aligned}
$$

It can be shown that this Toeplitz matrix decomposes as

$$
\begin{aligned}
& \mathbf{T}_{1 \mid i}^{\mathrm{ref}}
\end{aligned}
$$

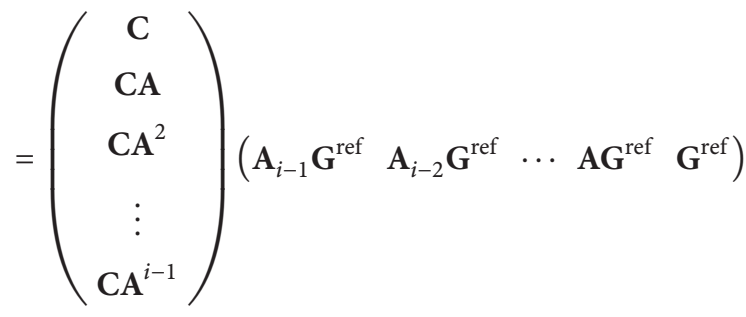

$$
\begin{aligned}
& =\mathbf{O}_{i} \mathbf{C}_{i}^{\mathrm{ref}} \text {, }
\end{aligned}
$$

where $\mathbf{A}$ is the discrete state matrix and $\mathbf{C}$ is the discrete output matrix. Therefore, the Toeplitz matrix can be based on to estimate the observability $\mathbf{O}_{i}$ and reference-reserved controllability matrix $\mathbf{C}_{i}^{\text {ref }}$ by applying the singular decomposition to it:

$$
\mathbf{T}_{1 \mid i}^{\mathrm{ref}}=\mathbf{U S V}^{T}=\left(\mathbf{U}_{1} \mathbf{U}_{2}\right)\left(\begin{array}{cc}
\mathbf{S}_{1} & \mathbf{O} \\
\mathbf{O} & \mathbf{O}
\end{array}\right)\left(\begin{array}{c}
\mathbf{V}_{1}^{T} \\
\mathbf{V}_{2}^{T}
\end{array}\right)=\mathbf{U}_{1} \mathbf{S}_{1} \mathbf{V}_{1}^{T},
$$

in which only the significant nonzero singular values $\boldsymbol{S}_{1}$ are reserved and those close to zeros due to noise influences are ignored, leading to

$$
\begin{aligned}
\mathbf{O}_{i} & =\mathbf{U}_{1} \mathbf{S}_{1}^{1 / 2}, \\
\mathbf{C}_{i}^{\text {ref }} & =\mathbf{S}_{1}^{1 / 2} \mathbf{V}_{1}^{T} .
\end{aligned}
$$

Based on (9), the system matrix $\mathbf{C}$ can be constructed to be the first $l$ rows of $\mathbf{O}_{i}$ and $\mathbf{G}^{\text {ref }}$ is the last $r$ columns of $\mathbf{C}_{i}^{\text {ref }}$. Consequently, the system matrix $\mathbf{A}$ can be found by using another shifted block Toeplitz matrix through

$$
\mathbf{A}=\mathbf{S}_{1}^{-1 / 2} \mathbf{U}_{1}^{T} \mathbf{T}_{2 \mid i+1}^{\mathrm{ref}} \mathbf{V}_{1} \mathbf{S}_{1}^{-1 / 2}
$$

which is due to the relationship of $\mathbf{T}_{2 \mid i+1}^{\mathrm{ref}}=\mathbf{O}_{i} \mathbf{A} \mathbf{C}_{i}^{\mathrm{ref}}$. This means that the system matrices $\mathbf{A}, \mathbf{C}$ in (1) can be recovered using the output covariance data matrices. However, in practice, the data length $j$ is limited and, therefore, the covariances expressed in (6) are just estimates. This means that these estimates may vary from measurements to measurements when data contains inevitable nonstationary contents, which will result in uncertainties in identification results. Moreover, when the SNR of measurements is low, the identification results may not be convergent.

In addition, because of the influences of different noises, the higher singular values in (8) are not zeros, which makes it difficult to identify the system order $n$. Therefore, it is often necessary to use a stabilization diagram to determine $n$ and corresponding model parameters.

Conventionally, the stabilization diagram is constructed by the increments of system order $n$ at a fixed row number. However, recent studies $[10,14,15]$ show that the efficiency and accuracy of identification also depend on the variation of the row number $i$ of the Hankel matrix and hence lead to an alternative stabilization diagram that is formed by consecutive increments of the row number $i$ at a fixed order $n$ and show more effectiveness, compared with the conventional one. However, it needs to specify $n$ first which is usually unknown for most applications. To overcome this shortage, this study constructs the alternative stabilization diagram by varying the order $n$ simultaneously at each increment $i$. This then ensures that significant modes can be selected automatically without the need to specify $n$ in advance.

\subsection{Eigen Realization Algorithm with Data Correlation} (ERA/DC). However, in implementing Cov-SSI, as the data length $j$ is limited, the covariances expressed in (5) are just estimates. It means that these estimates may vary from measurements to measurements. Especially when data contains inevitable nonstationary effects and high noise contents, identification results will have high uncertainties. To overcome these shortages, many researches have been made over year. Of particular interest are the ERA based approaches as they can produce superior identification results with both the conventional impulse response data [16] and the latest correlation data induced by white noise inputs [17].

As shown in [18], the correlation signal between two responses from white noise excitations is equivalent to the impulse responses of a dynamic system. It means that the correlation signal can be used directly for the time domain based identification methods. In $[19,20]$, the correlation signals were employed to construct the block Hankel matrix in implementing the standard ERA developed based on impulse inputs, rather than a white noise. These show that, from the scheme of ERA based identification, matrix in (5) 
can be viewed as the impulse responses, that is, the Markov parameters in ERA. In this way, Cov-SSI is the same as ERA and the improvements made on ERA can be applied to CovSSI.

To reduce noise influences, Juang et al. [21] improve ERA by using correlation data of impulse responses and named it as ERA with data correlation (ERA/DC). As shown in ERA/DC, the correlation data of the block Hankel matrix constructed from impulse responses also consists of the information of system matrix. It means that a further correlation of the correlation matrix of (5) can be calculated by (11) for system identifications:

$$
\mathbf{T} \mathbf{T}_{q}=\mathbf{T}(q) \mathbf{T}(0)^{T} \in \mathbf{R}^{l i \times l i},
$$

where the zero-lag correlation $\mathbf{T}(0)=\mathbf{Y}_{p}^{\mathrm{ref}} \mathbf{Y}_{p}^{\mathrm{ref}^{T}}$. According to this relationship and the way of constructing (5), a new block Toeplitz matrix can be constructed from the further correlation data and named as the further correlation matrix, which can be expressed as

$$
\begin{aligned}
\mathbf{U}_{1 \mid i}^{\mathrm{ref}}(q) & =\left(\begin{array}{cccc}
\mathbf{T T}_{q+1} & \mathbf{T T}_{q+2} & \cdots & \mathbf{T T}_{q+b} \\
\mathbf{T T}_{q+2} & \mathbf{T T}_{q+3} & \cdots & \mathbf{T T}_{q+b-1} \\
\cdots & \cdots & \cdots & \cdots \\
\mathbf{T T}_{q+a} & \mathbf{T T}_{q+a-1} & \cdots & \mathbf{T T}_{q+a+b}
\end{array}\right) \\
& \in \mathbf{R}^{a l i \times b l i} .
\end{aligned}
$$

As this block Toeplitz matrix also contains the information of system matrix in the format of (6), the SVD process expressed in (4) can be applied to it to estimate the system matrix A and subsequently calculate modal parameters using (4). However, as the effect of more noise reduction and possible nonstationary suppression due to the further correlation operations, this further correlation matrix based SSI will give more accurate results, which will be evaluated in the next section.

\subsection{Average Correlation Signal Based Covariance Driven} Stochastic Subspace Identification (Acs-Cov-SSI). To reduce the deficiency of in implementing Cov-SSI/ref, the correlation signals between sensors are taken as the input. Moreover, the correlation signal can be considered as the free-vibration decay or the impulse responses of a dynamic system [12] and has been used in various time domain based identification methods.

The correlation signals are equivalent to the impulse responses whose spectra are infinitely wide in theory. Thus, they can be taken unsurprisingly to be the output of statespace equation corresponding to white noise excitations, which meets the fundamental assumption when SSI scheme is developed. Subsequently, a Hankel data matrix as expressed in (5) can be constructed using the correlation signals, rather than the raw response signals. In the same way, their covariance data matrixes of correlation signals also have the form of (7). Therefore, a correlation signal based Cov-SSI/ref can be implemented for SSI.
However, the correlation signals estimated from one data record are often not sufficiently effective to suppress the noise and nonstationary influences when their contents are very high, such as that of the vibration responses of the truck frame presented in Section 2.1. Usually, there are multiple data records available which may be collected under similar or different operating conditions. Thus, it is natural to combine these records together for obtaining a more reliable identification result. As correlation signals can be calculated using a specified reference sensor for all different records, the phase information between different records can be preserved by these reference-based correlation signals and hence an average of the correlation signals can be performed between different data records. The average will thus enhance the contents with regular or periodic components by suppressing the irregular random contents in different data records. Particularly, the component that associates with one of the system modes is often the more significant one. Moreover, its autocorrelation signal of the reference sensor always has a zero-phase in different data records. Therefore, the average of the autocorrelation signals from different records effectively improves the SNR of the resultant correlation signals. Simultaneously, as cross-correlation signals maintain the relative phase connections to the reference signals, the average also enhances the desired regular components and suppresses the noise contents. Specifically, the average correlation signals can be obtained by the following key steps:

(1) Obtain $k$ numbers of data segments from $l$ channels measurements either by using multiple measurement records or by segregating a very long record into small ones.

(2) Select a reference channel such as $p$ which may have better SNR through a spectrum analysis or an analytical analysis to estimate which of the sensors is less influenced by interferences. For instance, as the engine and power train may cause more influences on the sensors in the front of the frame, one of the sensors which is at rear can be used as the reference.

(3) Calculate the auto- and cross-correlation signals of each segment with $N$ samples for different channels $i=1,2, \ldots, l$ when taking the channel $p$ as the reference channel. Consider

$$
r^{i p}(\tau)=\frac{1}{N-i} \sum_{q=0}^{N-q-1} y^{i}(q+\tau) y^{p}(q)
$$

which can be calculated using the fast Fourier transform (FFT) algorithm to improve overall identification efficiency.

(4) Average the correlation signals from different segments to obtain the average correlation signals for corresponding channels:

$$
\overline{r^{i p}(\tau)}=\frac{1}{k} \sum_{k=1}^{k} r_{k}^{i p}(\tau)
$$


TABLE 1: Parameters in the FEM model.

\begin{tabular}{lccccc}
\hline Model & Elastic modulus $(\mathrm{MPa})$ & Poisson's ratio & Unit & Value of unit $(\mathrm{mm})$ & Comment \\
\hline Longitudinal beams & $2.1 \times 10^{5}$ & 0.3 & Shell 63 & 30 & Two symmetrical side beams \\
Horizontal beams & $2.1 \times 10^{5}$ & 0.3 & Shell 63 & 15 & Five horizontal beams \\
Linking board & $2.1 \times 10^{5}$ & 0.3 & Shell 63 & 15 & Four link boards for each horizontal beam \\
Joints & $2.1 \times 10^{5}$ & 0.3 & Combine 14 & 16 & Diameter values \\
\hline
\end{tabular}

It will show that using the correlation signal and its average will significantly improve the SNR of input data and result in more accurate, robust, and efficient identification.

\section{Verification of the Average Correlation Signal Based SSI}

To verify the suggested method, two offline tests were conducted when the frame was at free status. The first one is a standard hammer test in which both inputs and outputs are used for modal identification by the commercial software. The second one is a random hammer test in which only the outputs are used to determine the modal parameters by the proposed Acs-Cov-SSI method. In addition, FEM was also used to calculate the modes for further benchmarking the test results.

3.1. Modal Characteristics from a Standard Hammer Test. During the standard hammer test, both the input hammer force and the vibration responses at points shown in Figure 1(a) are recorded and the modal parameters are determined using the commercial software provided with a PolyLSCF method abbreviated from the multireference point based least square method in the complex frequency domain. The test needs to be performed particularly carefully in order to reduce errors due to inadequate hammering points, hitting amplitudes, and force windowing and using an oversampling mechanism for logging the force signal. The HyperWorks software was used in the FEM in which the frame in Figure 1(a) was modelled with 54503 units consisting of 27 basic components. Table 1 depicts key parameters in FEM. From the grid arrangement and necessary calculation parameters, first ten vibration modes in the low frequency range below $100 \mathrm{~Hz}$ are obtained by HyperWorks software.

Figures 3 and 4 present the results for the measured and FEM predicted modes, respectively. It can be seen that both the modal shapes and the frequency values for modes at 10.20 Hz, 26.44 Hz, 32.86 Hz, $34.90 \mathrm{~Hz}, 52.12 \mathrm{~Hz}$, and $75.91 \mathrm{~Hz}$ from the measurement agree closely with corresponding predictions at $9.12 \mathrm{~Hz}, 25.45 \mathrm{~Hz}, 34.00 \mathrm{~Hz}, 35.85$, and $76.63 \mathrm{~Hz}$, respectively. However, because of inevitable errors in both the measurement and calculation due to noise influences, sensor mounting uncertainties, and the inaccuracy of modelling the joints, the modal shapes measured at $40.71 \mathrm{~Hz}, 46.59 \mathrm{~Hz}$, $70.78 \mathrm{~Hz}, 80.36 \mathrm{~Hz}, 85.81 \mathrm{~Hz}$, and $95.59 \mathrm{~Hz}$ cannot agree very well with the corresponding predicted ones, even though several of them show very close frequency values. In addition, the differences between these two methods also indicate that the FE analysis may not be very accurate because of the inaccuracy in modelling the complicated joints. Nevertheless, as most of frequency values are close to each other between the measurement and prediction, the results are acceptable as benchmarks for the proposed method.

3.2. Modal Characteristics of a Random Hammer Test with Acs-Cov-SSI. During the random hammer test, four hammers with rubber heads hit the frame at points $3,4,9$, and 10 simultaneously, but with a random manner, as the continuous hits were achieved by four different people running the hammer with different forces and different rates. In this way, the excitations produced are similar to the situation when the vehicle travels on unpaved roads. During the continuous impacts, the 12 acceleration responses are recorded at $1000 \mathrm{~Hz}$ for 10 times, with each lasting for 100 seconds, in order to achieve sufficient averages for obtaining good correlation signals in implementing the Acs-Cov-SSI.

As shown in Figure 5, the short segments of acceleration responses at sensors 3, 4, 5, and 6 exhibit clear nonstationary phenomena due to the impacts. However, their noise contents are relatively low compared with the signals in Figure 1, as their spectra show distinctive peaks due to modal responses. To suppress the nonstationary and noisy effects, each data record is divided into 10 equal segments after applying a low pass filtering with a $100 \mathrm{~Hz}$ cutoff frequency. Thus, the averages for correlation signal calculation can be up to 100 times, allowing sufficient noise suppression.

Figure 6 shows the results from Acs-Cov-SSI, the results from Acs-Cov-SSI was obtained when the length of correlation signal is 1024 points covering at least two periods of the lowest modes. From the stable frequency centers shown in the alternative stabilization diagram in Figure 6(a), it can be observed that more than 12 modes can be extracted based on the stable modes across the row increment. Firstly, the modes at the potential frequency bin of $\pm 0.5 \mathrm{~Hz}$ are selected to be candidates when the occurrence rate across the last 30 rows is more than $50 \%$. Then, these candidates are further refined by keeping only those of their modal assurance criterion (MAC) [22] and damping ratio values within 0.1 and \pm 0.1 , respectively. Finally, the mean values of frequencies, damping ratios, and modal shapes from the selected modes are taken as the identification results.

Figure 6(b) presents the results obtained from the random hammer test. By comparing the results with that of Figure 3 obtained by the standard hammer tests, it can be found that, apart from the two rigid vibration modes at $2.363 \mathrm{~Hz}$ and $3.282 \mathrm{~Hz}$, the remaining 12 modes are consistent with those of the standard hammer test in that their modal shapes and frequency values are very close to each other, as detailed in Table 2. However, the damping values obtained 
TABLE 2: Comparison of modal parameters between standard hammer test and random hammer test.

\begin{tabular}{lccccccccccccc}
\hline Modal number & 1 & 2 & 3 & 4 & 5 & 6 & 7 & 8 & 9 & 10 & 11 & 12 \\
\hline Frequency (Hz) & 0.46 & 0.21 & 1.75 & 1.06 & 0.69 & 0.81 & 0.90 & 0.84 & 0.25 & 0.74 & 0.68 & 0.66 \\
\hline Damping ratio (\%) & -0.50 & -0.76 & -0.33 & 0.02 & -0.68 & 0.03 & -1.04 & 0.39 & -0.11 & -0.27 & 0.11 & -0.37 \\
\hline MAC & 0.01 & 0.01 & 0.17 & 0.01 & 0.02 & 0.10 & 0.21 & 0.15 & 0.11 & 0.20 & 0.02 & 0.06 \\
\hline
\end{tabular}

$10.20 \mathrm{~Hz}, 1.95 \%$

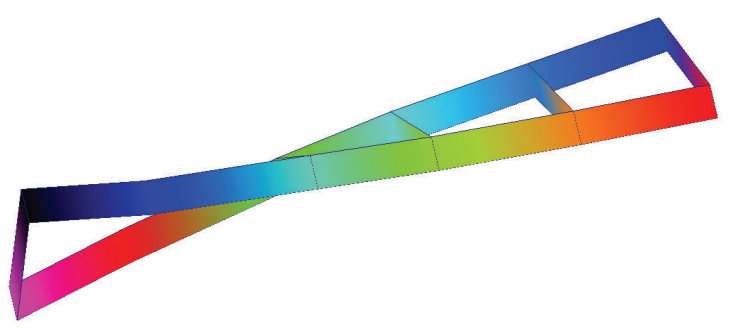

$32.86 \mathrm{~Hz}, 1.56 \%$

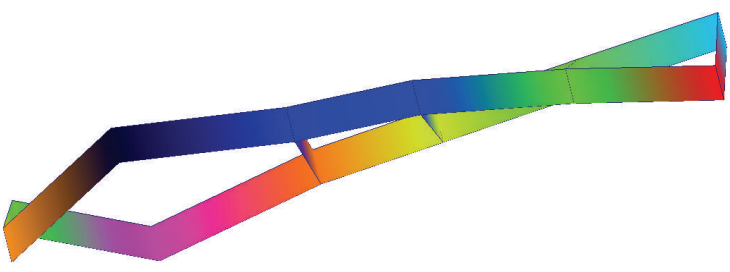

$40.71 \mathrm{~Hz}, 1.47 \%$

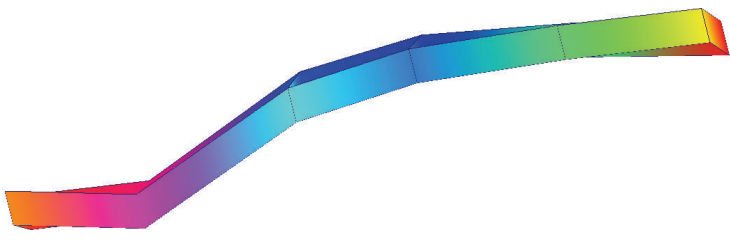

$52.12 \mathrm{~Hz}, 0.66 \%$

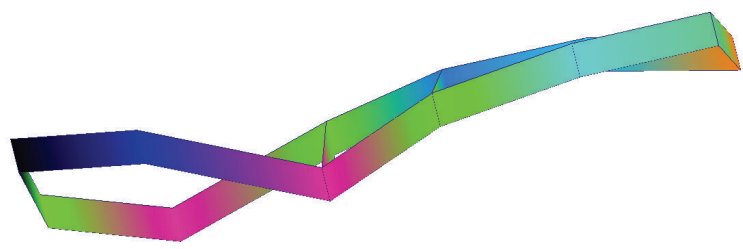

$75.91 \mathrm{~Hz}, 0.22 \%$

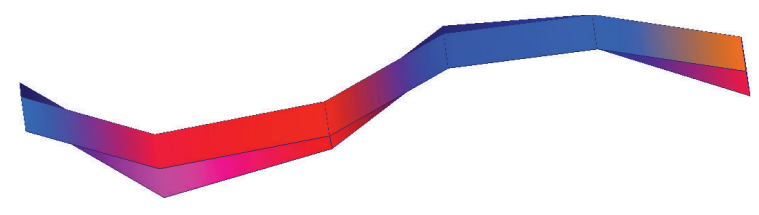

$85.81 \mathrm{~Hz}, 1.16 \%$

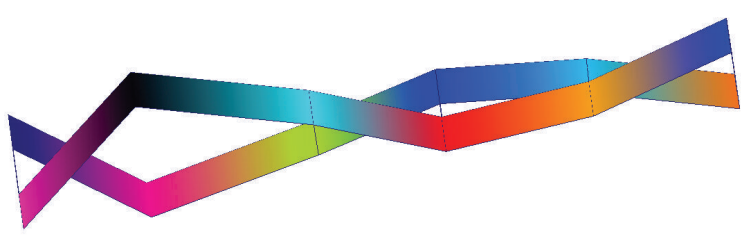

$26.44 \mathrm{~Hz}, 0.78 \%$

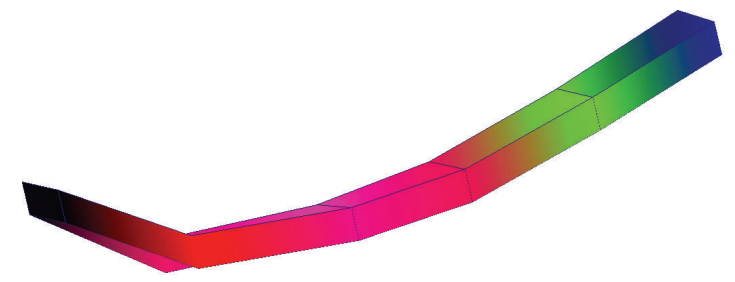

$34.90 \mathrm{~Hz}, 1.18 \%$

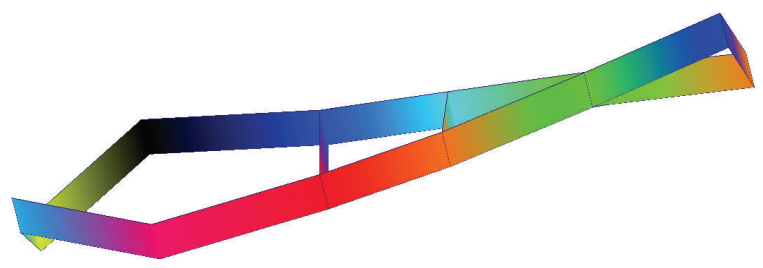

$46.59 \mathrm{~Hz}, 1.53 \%$

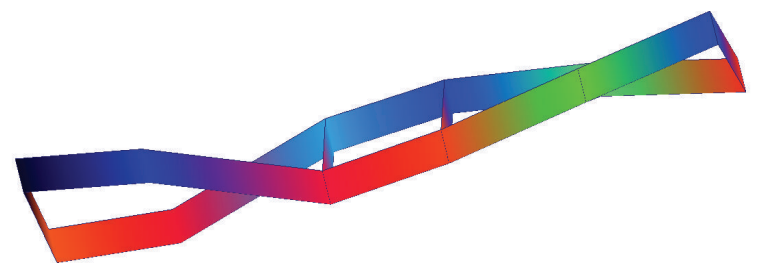

$70.78 \mathrm{~Hz}, 1.53 \%$

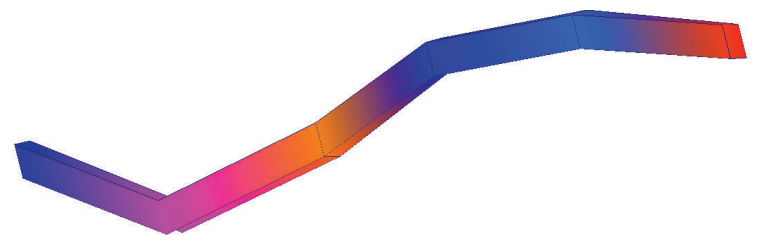

$80.36 \mathrm{~Hz}, 0.44 \%$

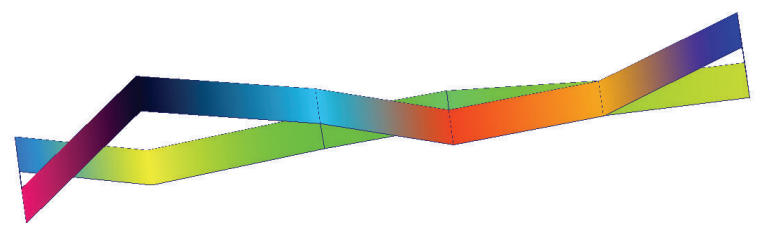

$95.59 \mathrm{~Hz}, 0.12 \%$

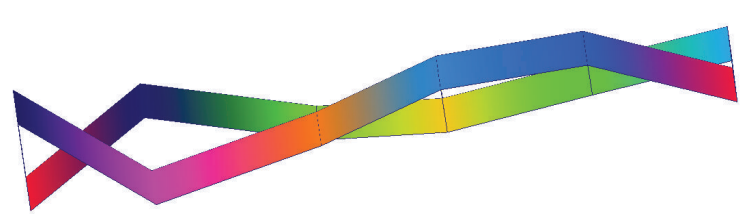

FIGURE 3: Modal identification results by the standard hammer test using the commercial software. 

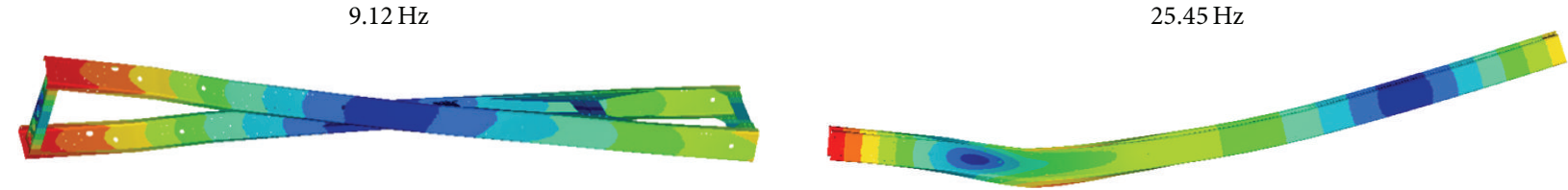

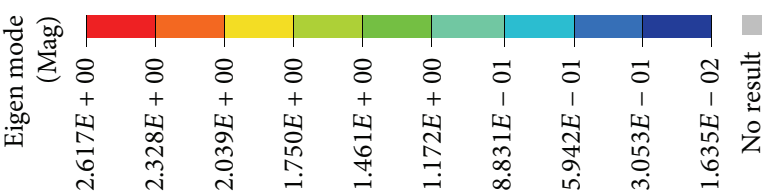

$34.00 \mathrm{~Hz}$
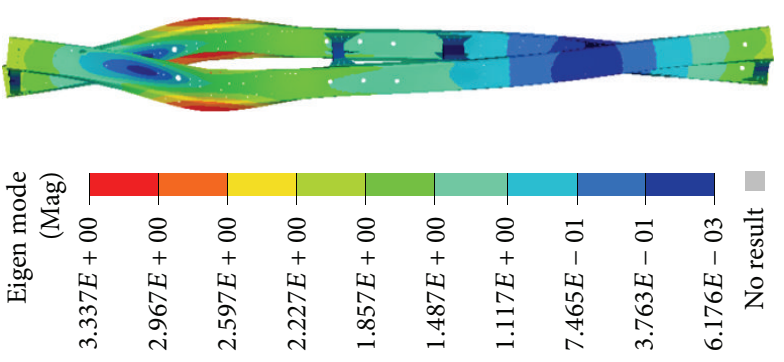

$45.93 \mathrm{~Hz}$
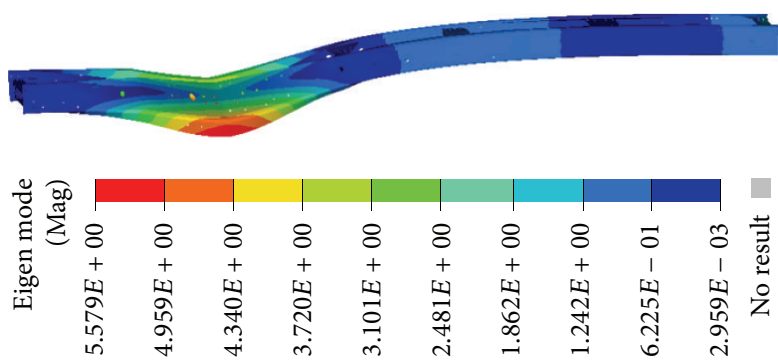

$51.94 \mathrm{~Hz}$
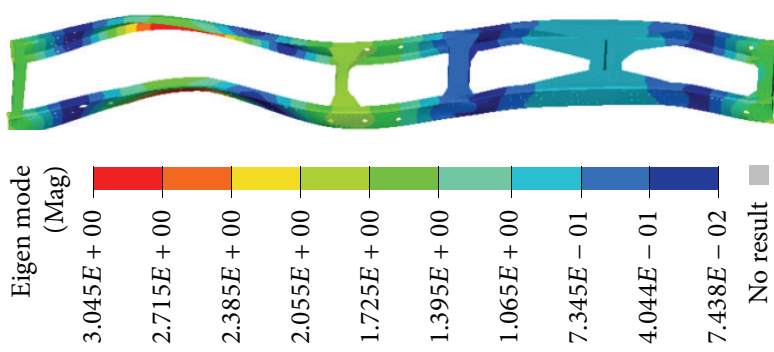

Not identified

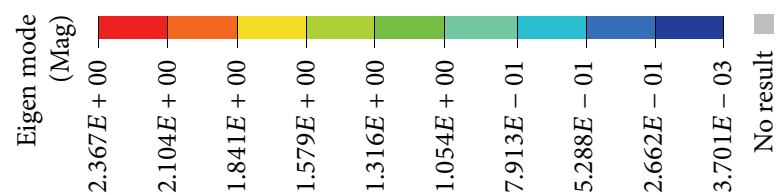

$35.85 \mathrm{~Hz}$
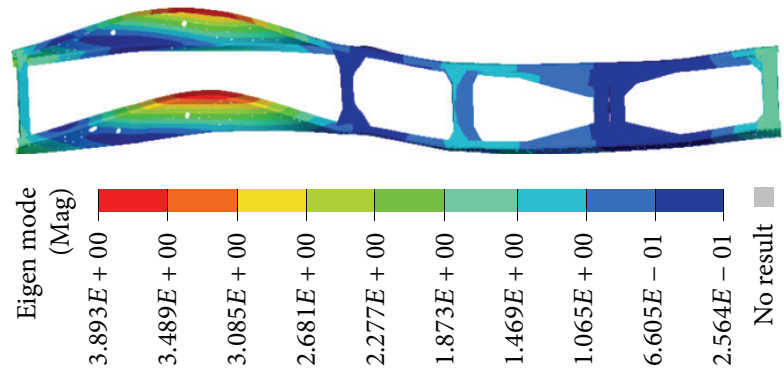

$50.30 \mathrm{~Hz}$

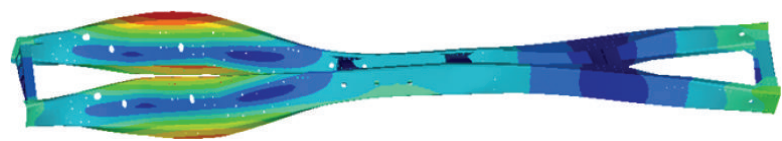

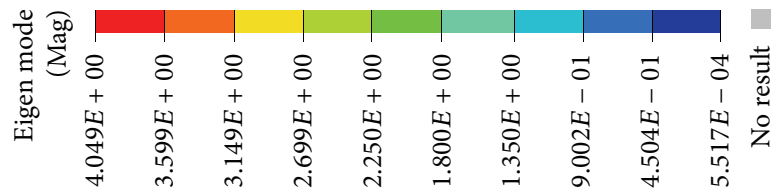

$76.63 \mathrm{~Hz}$

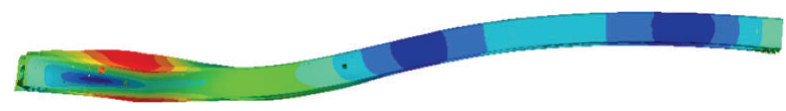

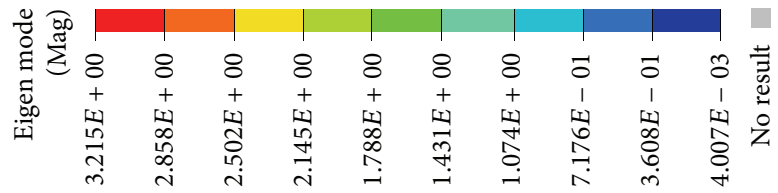

$93.07 \mathrm{~Hz}$

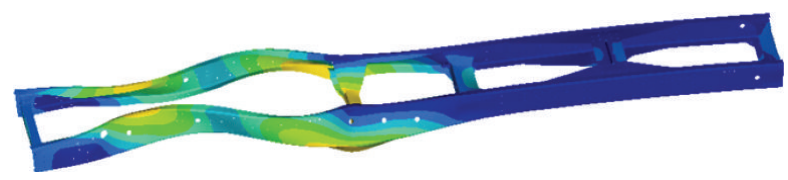

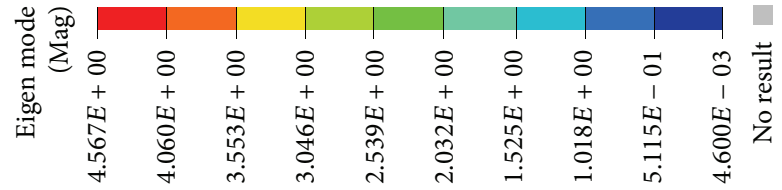

FIGURE 4: Modal parameters from FEM. 

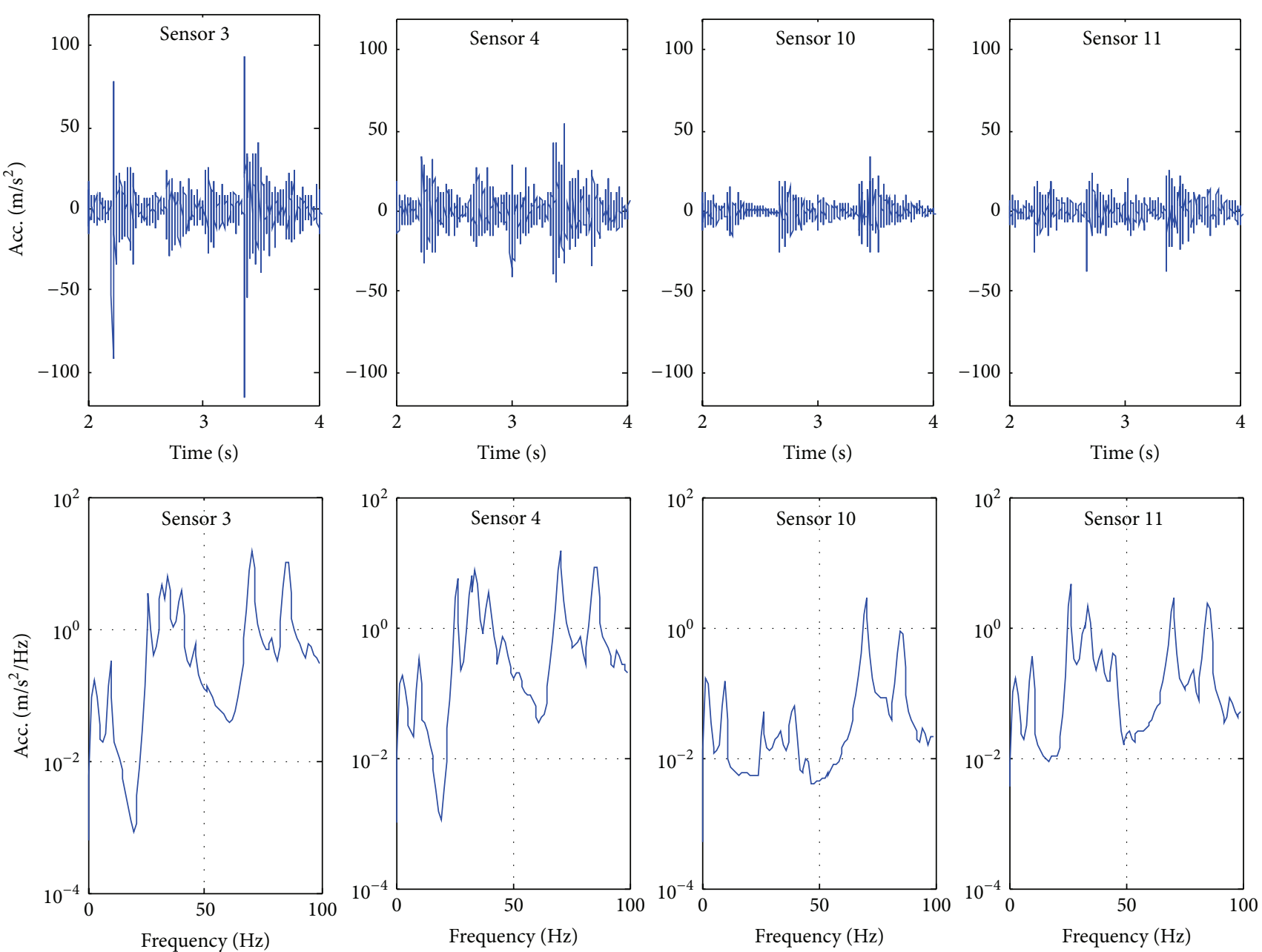

FIGURE 5: Examples of acceleration signals from the random hammer test.

from Acs-Cov-SSI are slightly higher. This difference may stem from the deficiency of damping ratio estimation with SSI based methods [15]. Nevertheless, the difference can be acceptable because the variation of damping ratio values is agreed upon between the two methods. Therefore, the test proves that the proposed method can achieve the same accuracy as that of the commercial one. However, the proposed method uses output-only data and with little restriction to excitations. It means that it can be easily implemented and hence particularly suitable for online identification.

Moreover, a comparison of stabilization diagrams is made between different averages applied to the correlation signals when the raw signals are added by more than $30 \%$ random noise. From the stabilization diagrams in Figure 7 it can be seen that the spurious modes due to noise become less with larger averages, resulting in a clearer diagram. In the meantime, the potential modes at $51 \mathrm{~Hz}$ and $65 \mathrm{~Hz}$ become more identifiable. This shows the high effectiveness of the average of correlation functions.

\section{Characterization of the Modal Responses to Different Roads}

The vehicle is designed to operate mainly in different development sites where the road condition is usually unpaved with very poor condition. Figure 8(b) shows an example of such roads on which current tests are based. The road has very oscillating profiles with many large valleys and sharp summits. Obviously, these severe road conditions will cause severe dynamic loads and high vibrations to the frame and also cause difficulties to handling the vehicle. To assess these effects, two road tests were carried out on both the roads with speed bumps and the roads without any pavements, respectively, as shown in Figures 8(a) and 8(b). The first one is to evaluate the frame dynamic responses when the vehicle passes successive bumps which induce extreme excitations. During this test, the vehicle accelerated to about $20 \mathrm{~km} / \mathrm{h}$ first. Then, its engine was shut down to let the vehicle slide across a number of consecutive bumps. As the bump produces high impacts and there are no engine excitations, the data will have good SNR for identification. 


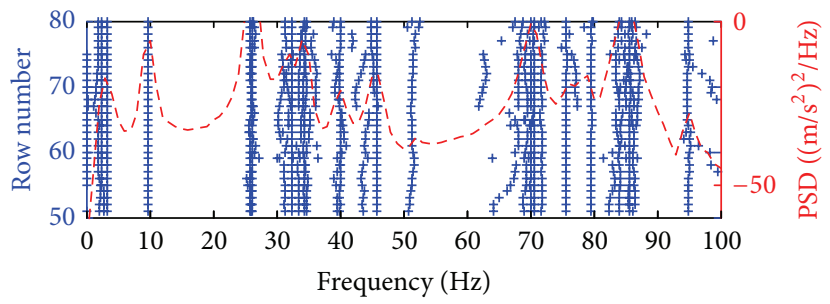

(a)
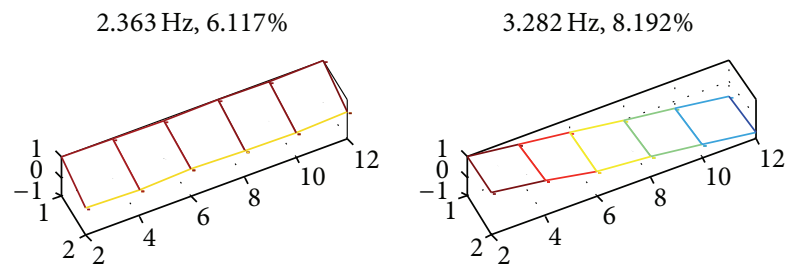

$9.732 \mathrm{~Hz}, 2.442 \%$
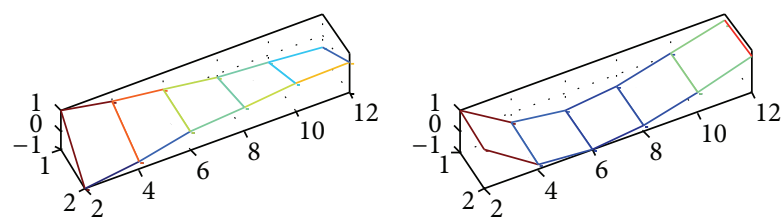

$31.1 \mathrm{~Hz}, 2.011 \%$

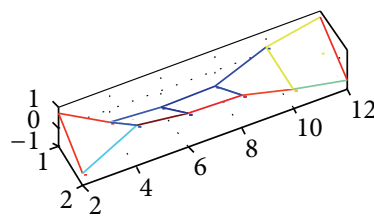

$34.81 \mathrm{~Hz}, 0.6947 \%$

$40.03 \mathrm{~Hz}, 2.279 \%$

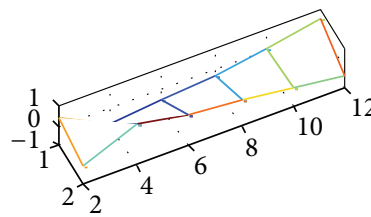

$71.81 \mathrm{~Hz}, 1.025 \%$

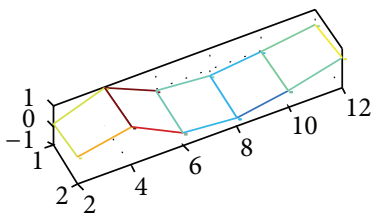

$45.78 \mathrm{~Hz}, 1.494 \%$

$51.18 \mathrm{~Hz}, 1.803 \%$
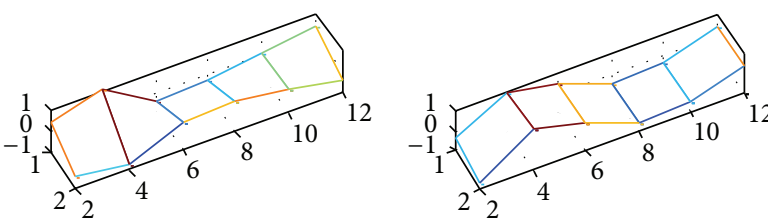

$86.19 \mathrm{~Hz}, 1.394 \%$

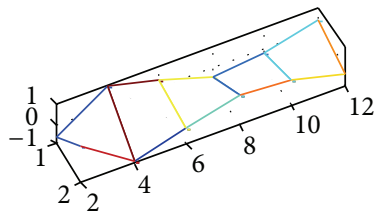

$75.67 \mathrm{~Hz}, 0.3361 \%$

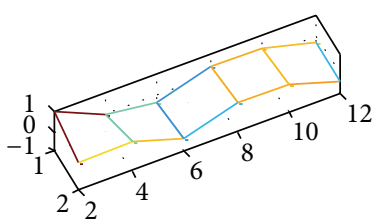

$79.62 \mathrm{~Hz}, 0.7111 \%$

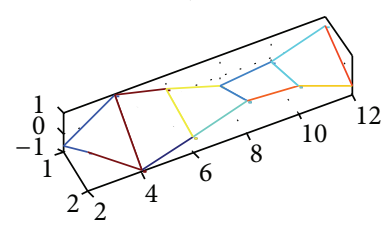

$94.92 \mathrm{~Hz}, 0.4934 \%$
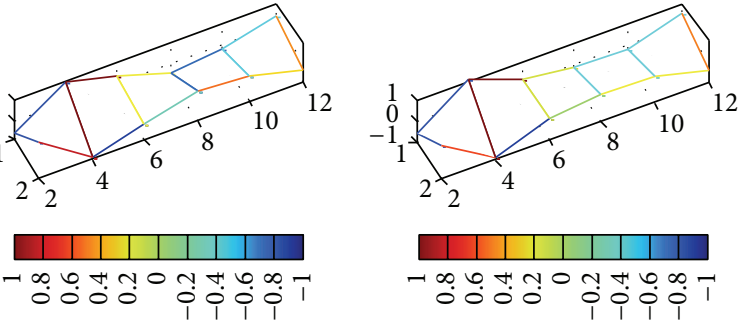

(b)

FIGURE 6: Modal identification results based on a random hammer test by Acs-Cov-SSI. (a) Stabilization diagram and (b) identified modes.

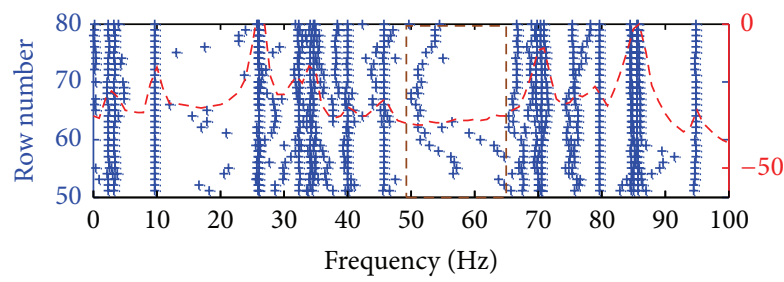

(a)

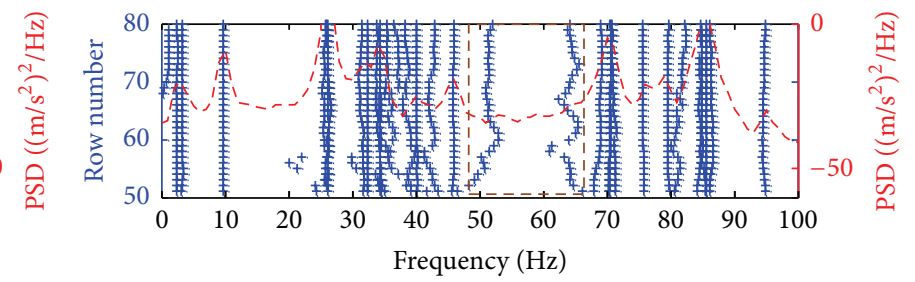

(b)

FIGURE 7: Comparison of stabilization diagrams for different averages. (a) 100 averages and (b) 140 averages. 


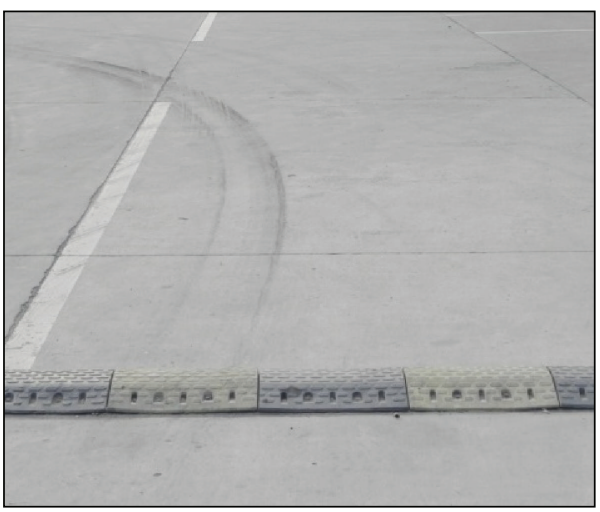

(a)

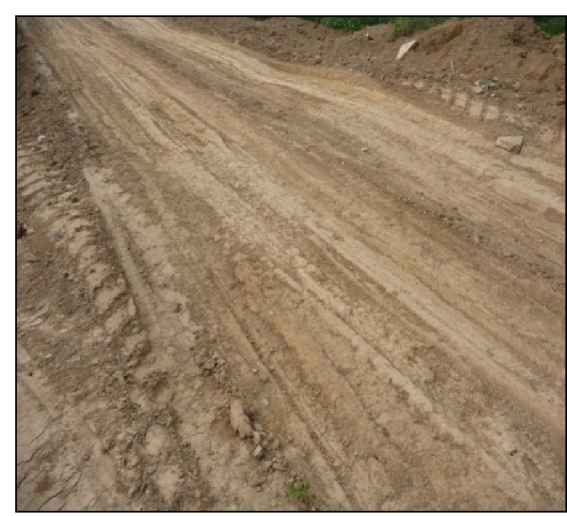

(b)

FIgURE 8: Illustration of the test road conditions. (a) Bumped road and (b) unpaved road.

During the unpaved road test, the vehicle travelled at about $20 \mathrm{~km} / \mathrm{h}$ for both loaded and unloaded cases, which are typical operating modes for such vehicles as they often repeat the same trips for transporting materials in-site.

For each test case, more than 10 data records with a sampling rate of $1000 \mathrm{~Hz}$ were obtained. Each record lasts about 6 minutes or 360,000 samples, yielding a sufficient data length of 1,800 for correlation signal calculation when segregating each record into 20 short segments. Consequently, the number of averages can be more than 100 in order to suppress noise influence effectively for more accurate mode extraction.

\subsection{Modal Characteristics on Bumped Road without Load.} Figure 9 shows the alternative stabilization diagram and corresponding identification results for the case when the vehicle travelled on the bumped road without load. As expected, a large number of modes can be identified because of the wide frequency excitations due to the large and sharp impacts induced by travelling across bumps. However, these modes behave very differently from that of the free frame presented in Figure 6.

Only one rigid mode at $3.772 \mathrm{~Hz}$ is shown up as the excitations between two sides are nearly the same when the vehicle crosses the bumps forward. Because the engine and loading system are mounted in the front of the frame, the mode exhibits a high pitch motion at the rear part as the container is not loaded and can be activated by impacts of the bump.

For the modes from the frame responses, the smooth shapes for the first twist mode and bending mode identified previously are not shown because of the constraints of different subsystem and parts installed onto the frame. Instead, six modes identified in the frequency range from $7.135 \mathrm{~Hz}$ to $40.87 \mathrm{~Hz}$, in which the spectrum amplitudes are high, exhibit local twist or bending profiles. However, according to the modal shapes, it can be deduced that high relative deformations of the frame occur at sensor positions of 3, 4, 5 , and 6. It indicates that the frame is subject to high dynamic loads at these positions and the stiffness at this part of the frame may need to be improved. In addition, it is worth noting that the modes at $7.135 \mathrm{~Hz}$ and $11.04 \mathrm{~Hz}$ may affect the handling stability as their frequency values are very low and close to the resonances of the tyres.

For the modes at frequencies higher than $45 \mathrm{~Hz}$, there are also little similarities with that of free frame status. Although the mode at $51.16 \mathrm{~Hz}$ shows close frequency and damping values to that of $51.18 \mathrm{~Hz}$, the two shapes are different because of the effect of additional joints on the frame. Moreover, the modes in this frequency range are associated with lower vibration energy, so they may induce less dynamic loads. However, their vibrations and associated noise can bring problems to ride comforts and environmental pollution.

From the above discussion, it can be concluded that travelling across bumps allows a good online identification of the modes associating with the frame. The result obtained online is very different from that obtained offline. In general, the online results show more modes below $45 \mathrm{~Hz}$ and modal frequencies are about $2 \mathrm{~Hz}$ lower compared with the offline results. However, the modal shapes are very different between the two methods. Moreover, the online results reflect the true dynamics of the frame and are the final references to assess the performance of the frame and the vehicle. Fortunately, the suggested method in the study allows the online identification to be implemented without any difficulties.

4.2. Modal Characteristics on Unpaved Roads without Load. When the vehicle travels on unpaved roads with layouts as illustrated in Figure 8(b), the road excitations are relatively more random between different wheels. Therefore, the three common rigid modes, pitch, rolling, and bounce of the vehicle, are more likely to be excited. As shown in Figure 10(b), the first three modes at $1.057 \mathrm{~Hz}, 3.201 \mathrm{~Hz}$, and $3.844 \mathrm{~Hz}$ just correspond to these motions, respectively.

As the unpaved roads cause smaller excitations, less significant modes can be excited and more spurious modes due to noise are shown in the stabilization diagram of Figure 10(a). However, it is still not difficult to extract the modes at $7.307 \mathrm{~Hz}, 11.15 \mathrm{~Hz}, 54.11 \mathrm{~Hz}$, and $63.93 \mathrm{~Hz}$ which show similar frequency and damping values to that of the bumped roads. In addition, their modal shapes are close to each other. The modes at $37.71 \mathrm{~Hz}$ and $57.96 \mathrm{~Hz}$ may correspond to those at $40.87 \mathrm{~Hz}$ and $63.88 \mathrm{~Hz}$, respectively, 


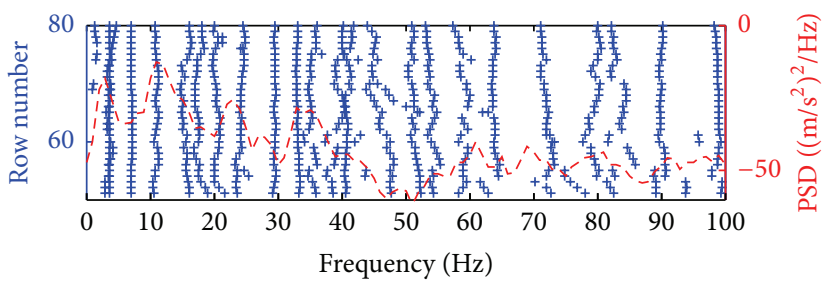

(a)

$3.772 \mathrm{~Hz}, 11.01 \%$

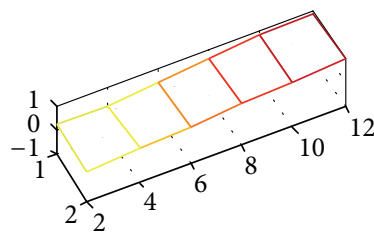

$29.63 \mathrm{~Hz}, 4.089 \%$

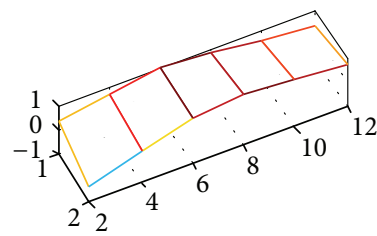

$53.97 \mathrm{~Hz}, 3.391 \%$

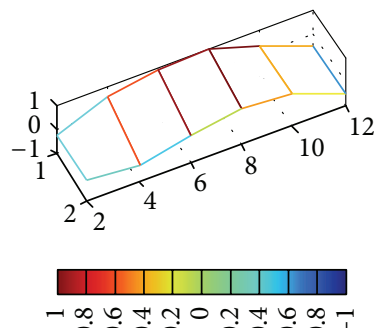

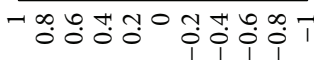

$7.135 \mathrm{~Hz}, 9.278 \%$

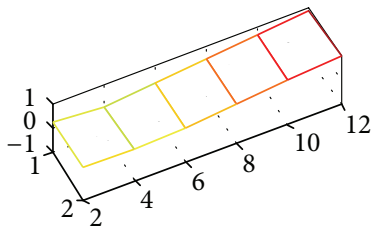

$33.11 \mathrm{~Hz}, 3.712 \%$

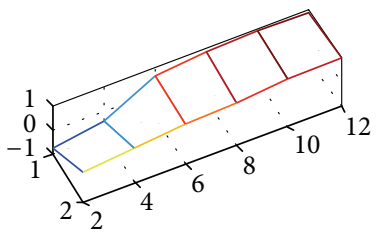

$63.88 \mathrm{~Hz}, 0.9667 \%$

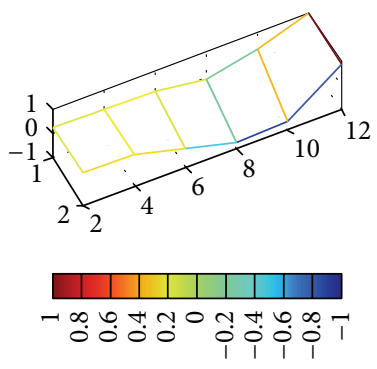

$11.04 \mathrm{~Hz}, 12.33 \%$

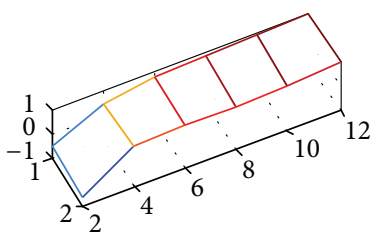

$40.87 \mathrm{~Hz}, 2.792 \%$

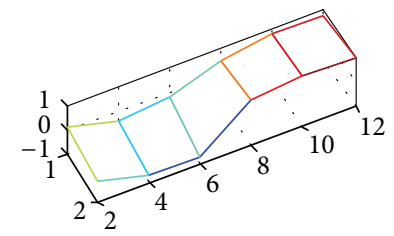

$89.95 \mathrm{~Hz}, 1.974 \%$
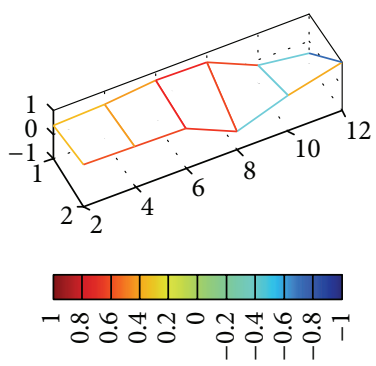

$24.1 \mathrm{~Hz}, 4.642 \%$

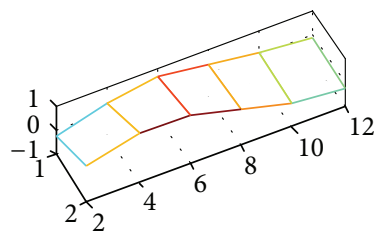

$51.16 \mathrm{~Hz}, 1.747 \%$

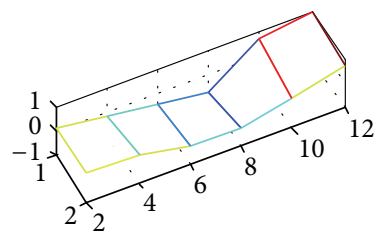

$98.94 \mathrm{~Hz}, 1.163 \%$

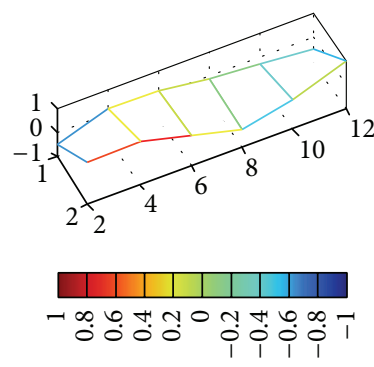

(b)

FIGURE 9: Modal characteristics of the frame on the bumped road without load. (a) Stabilization diagram and (b) identified modes.

as their modal shapes are very similar. However, the noise influences account for such differences in their frequency and damping values.

Nevertheless, these modal features have confirmed fundamental mode characteristics and their responses to road conditions, which in turn also verified again the proposed method. Moreover, all these modal shapes can indicate the high relative deformation positions and hence find the portion with high dynamic loads which provides references for structural improvement.

4.3. Modal Characteristics on Unpaved Roads with Load. When the vehicle is loaded, it travels more smoothly, which leads to less noise influences and hence a clearer stabilization diagram and more identifiable modes as shown in Figure 11.

However, the dynamic behaviour of the vehicle system, including the frame, changes significantly because of the loading mass which can be partly associated with the frame and alter the inherent modes. As shown in Figure 11, the identified modes for the loaded vehicle show significant differences from those of unloaded ones even though the road conditions are similar. A clear effect of the associated mass is the change of the bounce mode; as it can be seen in Figure 11(b), the rigid bounce occurs more severely at $2.004 \mathrm{~Hz}$, which is nearly $2 \mathrm{~Hz}$ lower compared with that of $3.844 \mathrm{~Hz}$ for the unloaded case. In addition, the damping value is significantly higher due to the nonlinear effect of the suspension system. However, the modes with more rolling at $3.114 \mathrm{~Hz}$ and $3.865 \mathrm{~Hz}$ are similar to the unloaded case in terms of the frequency and damping values.

Correspondingly, the effect of the associated mass also makes the structural responses of the frame occur at lower frequency values. Particularly, more modes in the lower frequency range start from about $5.834 \mathrm{~Hz}$ to $40 \mathrm{~Hz}$. Moreover, the modal shapes of these modes also show high relative deformation around sensor positions 3, 4, 5, and 6, indicating that this portion of the frame is subjected to high dynamic loads, similar to the unloaded cases. Therefore, the structure 


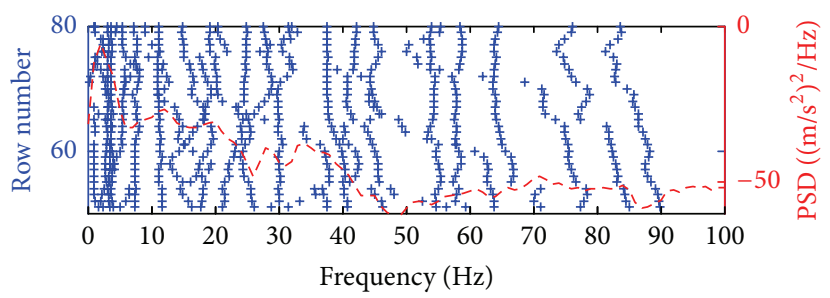

(a)

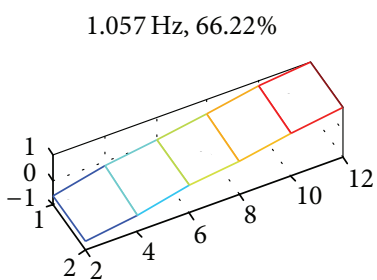

7.307 Hz, $19.29 \%$

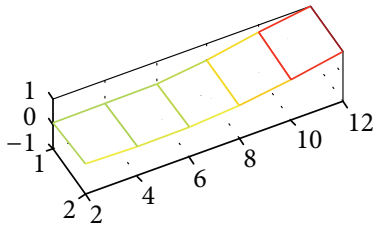

$54.11 \mathrm{~Hz}, 0.7006 \%$

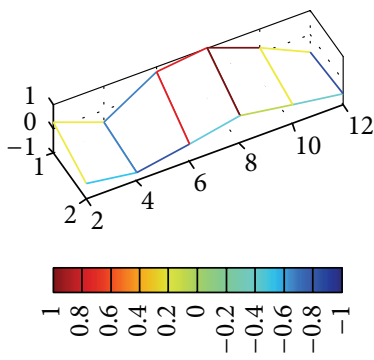

$3.201 \mathrm{~Hz}, 12.59 \%$

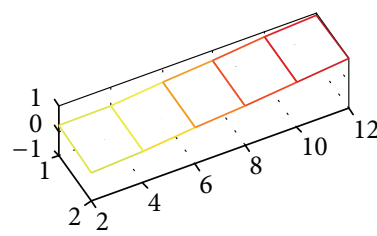

$11.15 \mathrm{~Hz}, 4.715 \%$

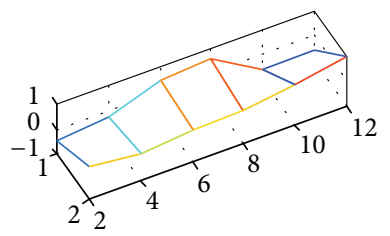

$57.96 \mathrm{~Hz}, 2.586 \%$

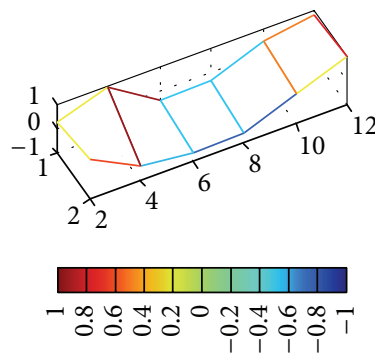

$3.844 \mathrm{~Hz}, 18.24 \%$

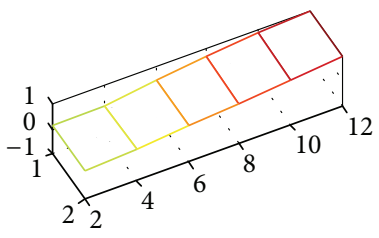

$37.71 \mathrm{~Hz}, 2.241 \%$

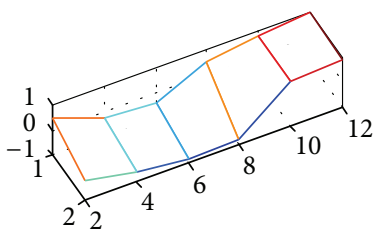

$63.93 \mathrm{~Hz}, 1.986 \%$

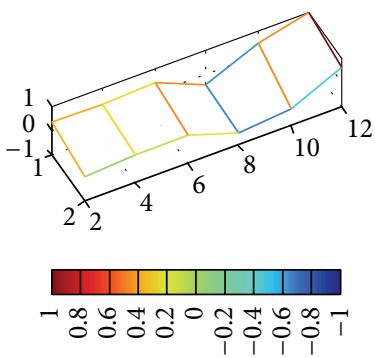

(b)

Figure 10: Modal characteristics of the vehicle frame on unpaved road at $20 \mathrm{~km} / \mathrm{h}$ without load. (a) Stabilization diagram and (b) identified modes.

of the frame should be further improved to enhance the stiffness in the parts. However, the mode at $11 \mathrm{~Hz}$ is not shown, indicating that there may be less interaction to the tyre systems and hence the brake performance can be maintained under loaded conditions.

In the higher frequency range, the modal shapes show combined twists and bends. However, as their corresponding spectral amplitudes are very low, they may not cause significant dynamic loads to the frame but add to ride discomfort. Particularly, all these modes show more oscillatory profiles in the front of the frame which can pass to the cab system easily.

\section{Conclusions}

In order to assess the dynamic performance of the frame in a heavy-duty dump truck, an online modal analysis is carried out using an improved SSI method. As the frame is subjected to high random excitations under the designed operating roads, the vibration responses exhibit and contain highly nonstationary and strong noise influences. These challenge the conventional SSI methods, such as the most promised reference-based covariance driven stochastic subspace identification (Cov-SSI/ref), to produce a convergent result. However, the proposed average correlation signals based CovSSI/ref can suppress these influences effectively and allow an efficient and reliable modal parameter identification.

By offline verification, the proposed method can produce identification results that are consistent with those from the commercial software using both the input and output measurements. Moreover, as the proposed method is more robust to noise influence, less demand for excitation conditions, and only relies on output responses, it can be easily realized for the online mode identification of the truck frame when it is integrated with full subsystems and when the vehicle operates under real operations. 


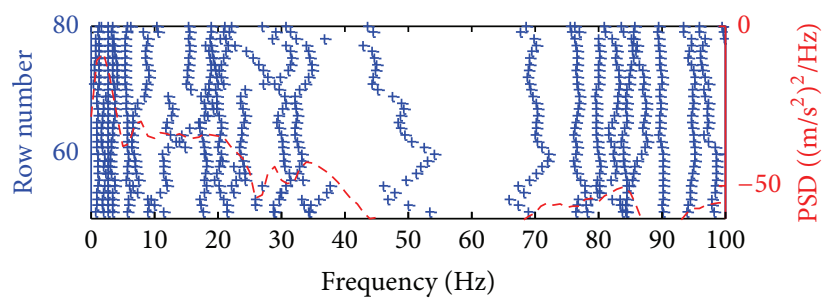

(a)

$1.051 \mathrm{~Hz}, 17.88 \%$

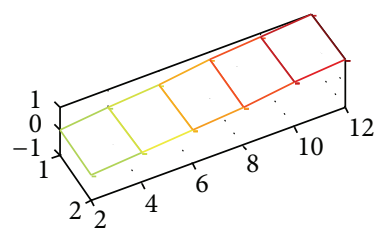

$5.834 \mathrm{~Hz}, 14.25 \%$

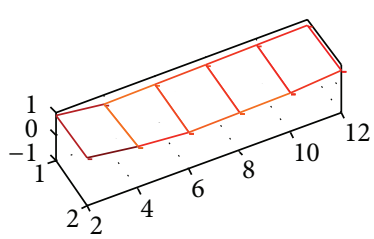

$2.004 \mathrm{~Hz}, 51.18 \%$

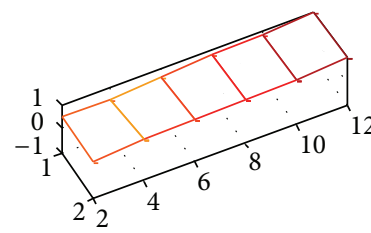

$18.05 \mathrm{~Hz}, 7.579 \%$

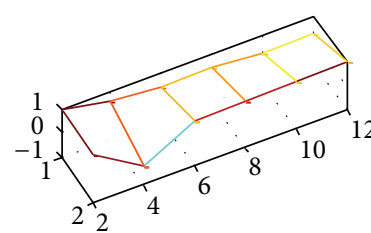

$3.114 \mathrm{~Hz}, 6.157 \%$

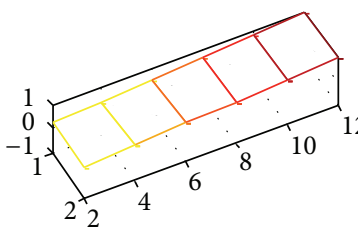

$76.78 \mathrm{~Hz}, 2.069 \%$

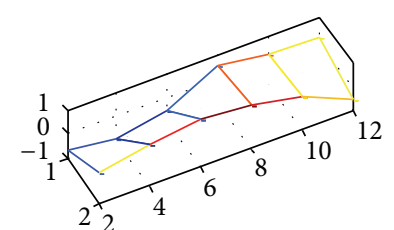

$3.865 \mathrm{~Hz}, 20.98 \%$

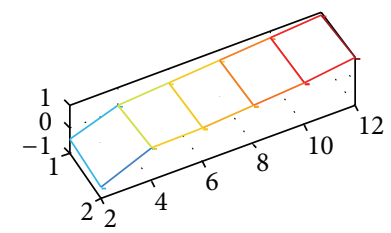

$79.96 \mathrm{~Hz}, 1.094 \%$

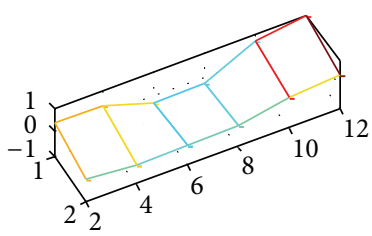

$83.01 \mathrm{~Hz}, 2.41 \%$

$89.97 \mathrm{~Hz}, 1.037 \%$

$94.92 \mathrm{~Hz}, 1.087 \%$
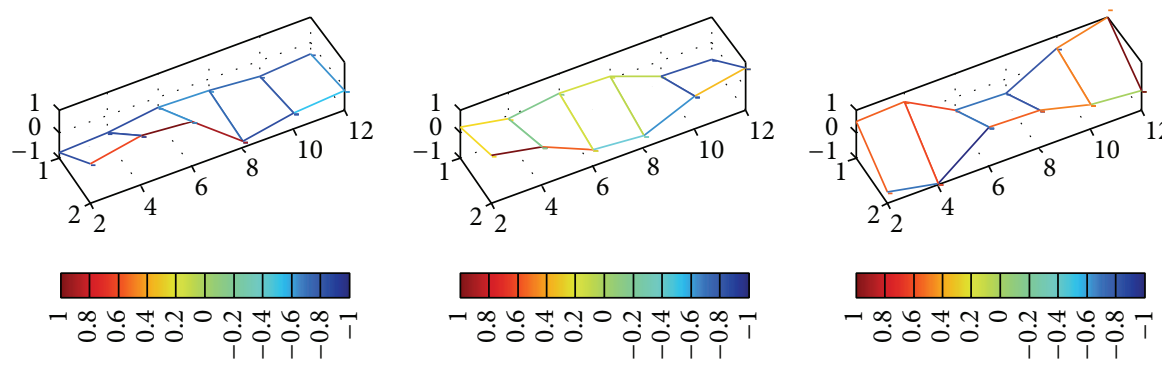

(b)

FIGURE 11: Modal characteristics of the vehicle frame on unpaved roads at $20 \mathrm{~km} / \mathrm{h}$ with load. (a) Stabilization diagram and (b) identified modes.

When the vehicle crosses successive speed bumps, the proposed method results in more identifiable modes because of the extreme excitations induced by the bumps. However, the mode number is still three less and modal parameters are significantly different compared with the offline results. The modal shapes from the offline results do not give clear indication of the part with high relative deformation. On the other hand, the online results clearly show that high relative deformation happens at sensor positions 3, 4, 5, and 6 where the frame is less rigged.

When it operates on the unpaved roads which are typical cases for various building and mining sites, the identifiable modes are less because of the smaller excitations on the roads. Without load, the frame shows about six modes but they can also indicate the high deformation position at the higher speed due to the higher excitations. However, when the vehicle is loaded, identifiable modes all shift to lower frequencies and the weak position can be also indicated by the modal shapes. In addition, more modes are identifiable because of less noise effect when the vehicle operates relatively smoother under the loaded condition. In addition, the modes at $7.135 \mathrm{~Hz}$ and $11.04 \mathrm{~Hz}$ may affect the handling stability as their frequency values are close to the resonances of the tyres.

Based on this successful online modal analysis, the performance of the proposed method has been proved. Moreover, it produces a realistic assessment of the dynamic characteristics of the frame and gives accurate locations where the frame needs to be improved further with higher rigidness.

\section{Conflict of Interests}

The authors declare that there is no conflict of interests regarding the publication of this paper. 


\section{Acknowledgments}

This research is supported by the Graduate Excellent Innovation Project of Shanxi Province of China with Grant no. 2012-302 and the High Technology Industrialization Project of Shanxi Province of China with Grant no. 2011-2368.

\section{References}

[1] C. Mi, Z. Gu, Q. Yang, and D. Nie, "Frame fatigue life assessment of a mining dump truck based on finite element method and multibody dynamic analysis," Engineering Failure Analysis, vol. 23, pp. 18-26, 2012.

[2] I. K. Yilmazçoban and Y. Kahraman, "Truck chassis structural thickness optimization with the help of finite element technique," The Online Journal of Science and Technology, vol. 1, no. 3, pp. 23-30, 2011.

[3] Z. Chen, T. Wang, and Z. Zhao, "The lightweight design of a dump truck frame based on dynamic responses," in Proceedings of the 18th International Conference on Automation and Computing, Paper no. 13072215, pp. 1-5, IEEE, Manchester, UK, September 2012.

[4] B. Peeters and G. De Roeck, "Stochastic system identification for operational modal analysis: a review," Journal of Dynamic Systems, Measurement and Control, vol. 123, no. 4, pp. 659-667, 2001.

[5] C. E. Ventura and M. T. Katherine, "Dynamic properties of a 32-storey building determined from different analysis methods of ambient vibration test data," in Proceedings of the 2nd International Operational Modal Analysis Conference, pp. 557564, Copenhagen, Denmark, May 2007.

[6] F. Magalhães, Á. Cunha, and E. Caetano, "Online automatic identification of the modal parameters of a long span arch bridge," Mechanical Systems and Signal Processing, vol. 23, no. 2, pp. 316-329, 2009.

[7] B. Peeters and G. De Roeck, "Reference-based stochastic subspace identification for output-only modal analysis," Mechanical Systems and Signal Processing, vol. 13, no. 6, pp. 855-878, 1999.

[8] E. Parloo, P. Verboven, P. Guillaume, and M. Van Overmeire, "Sensitivity-based operational mode shape normalisation," Mechanical Systems and Signal Processing, vol. 16, no. 5, pp. 757-767, 2002.

[9] E. Reynders and G. D. Roeck, "Reference-based combined deterministic-stochastic subspace identification for experimental and operational modal analysis," Mechanical Systems and Signal Processing, vol. 22, no. 3, pp. 617-637, 2008.

[10] G. Dong, J. Chen, and N. Zhang, "Investigation into on-road vehicle parameter identification based on subspace methods," Journal of Sound and Vibration, vol. 333, no. 24, pp. 6760-6779, 2014.

[11] X.-Q. Guan, M. Yuan, and J.-W. Zhang, "Application of subspace-based method in vehicle handling dynamic model identification and properties estimation," International Journal of Vehicle Design, vol. 56, no. 1-4, pp. 125-145, 2011.

[12] D.-Y. Chiang, C.-S. Lin, and F.-H. Su, "Identification of modal parameters from ambient vibration data by modified eigensystem realization algorithm," Journal of Aeronautics, Astronautics and Aviation, Series A, vol. 42, no. 2, pp. 79-86, 2010.

[13] M. Chang and S. N. Pakzad, "Modified natural excitation technique for stochastic modal identification," Journal of Structural Engineering, vol. 139, no. 10, pp. 1753-1762, 2013.
[14] Y. Zhang, Z. Zhang, X. Xu, and H. Hua, "Modal parameter identification using response data only," Journal of Sound and Vibration, vol. 282, no. 1-2, pp. 367-380, 2005.

[15] E. Reynders, R. Pintelon, and G. de Roeck, "Uncertainty bounds on modal parameters obtained from stochastic subspace identification," Mechanical Systems and Signal Processing, vol. 22, no. 4, pp. 948-969, 2008.

[16] J.-S. Lew, J.-N. Juang, and R. W. Longman, "Comparison of several system identification methods for flexible structures," Journal of Sound and Vibration, vol. 167, no. 3, pp. 461-480, 1993.

[17] D.-Y. Chiang and C.-S. Lin, "Identification of modal parameters from ambient vibration data using eigensystem realization algorithm with correlation technique," Journal of Mechanical Science and Technology, vol. 24, no. 12, pp. 2377-2382, 2010.

[18] C. R. Farrar and G. H. James III, "System identification from ambient vibration measurements on a bridge," Journal of Sound and Vibration, vol. 205, no. 1, pp. 1-18, 1997.

[19] B. A. Pridham and J. C. Wilson, "A study of damping errors in correlation-driven stochastic realizations using short data sets," Probabilistic Engineering Mechanics, vol. 18, no. 1, pp. 6177, 2003.

[20] D. M. Siringoringo and Y. Fujino, "System identification of suspension bridge from ambient vibration response," Engineering Structures, vol. 30, no. 2, pp. 462-477, 2008.

[21] J.-N. Juang, J. E. Cooper, and J. R. Wright, "Eigensystem Realization Algorithm using Data Correlations (ERA/DC) for modal parameter identification," Control-Theory and Advanced Technology, vol. 4, no. 1, pp. 5-14, 1988.

[22] R. J. Allemang, "The modal assurance criterion-twenty years of use and abuse," Sound and Vibration, vol. 37, no. 8, pp. 14-21, 2003. 

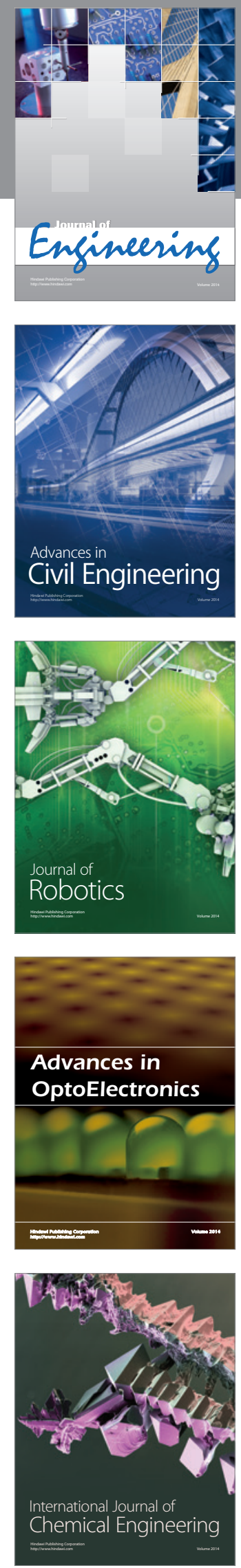

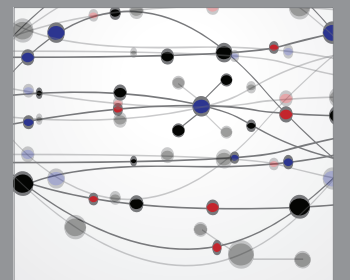

The Scientific World Journal
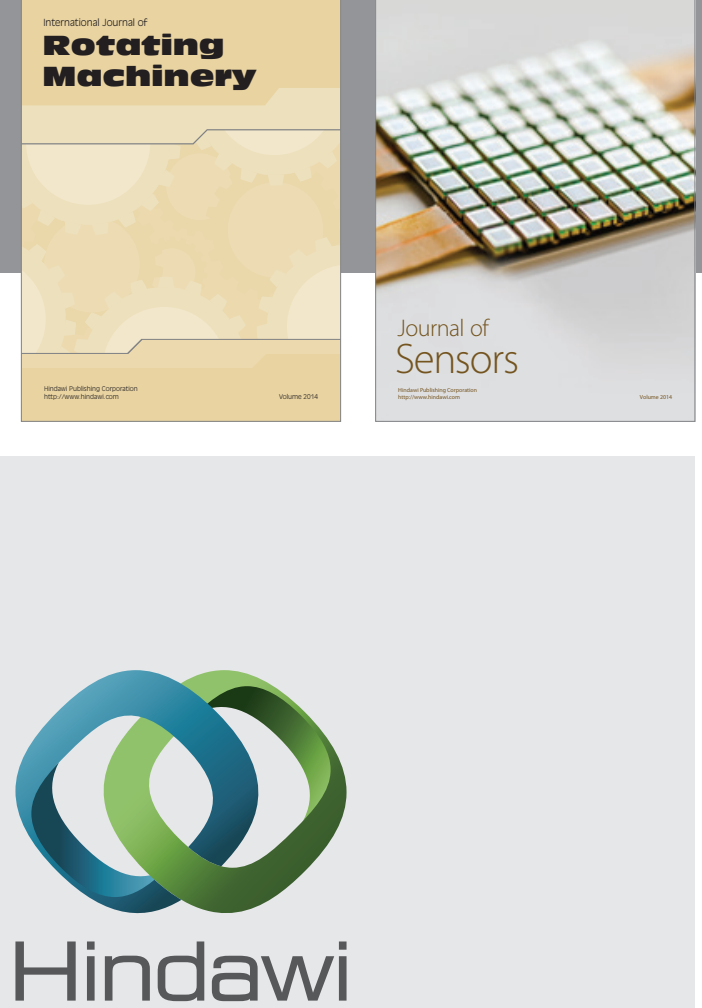

Submit your manuscripts at http://www.hindawi.com
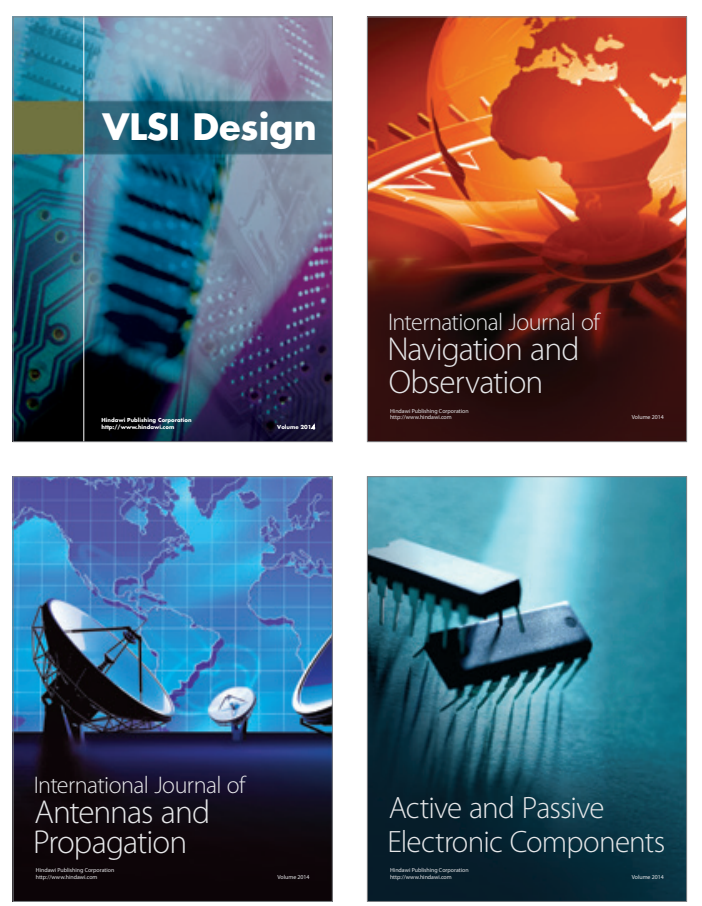
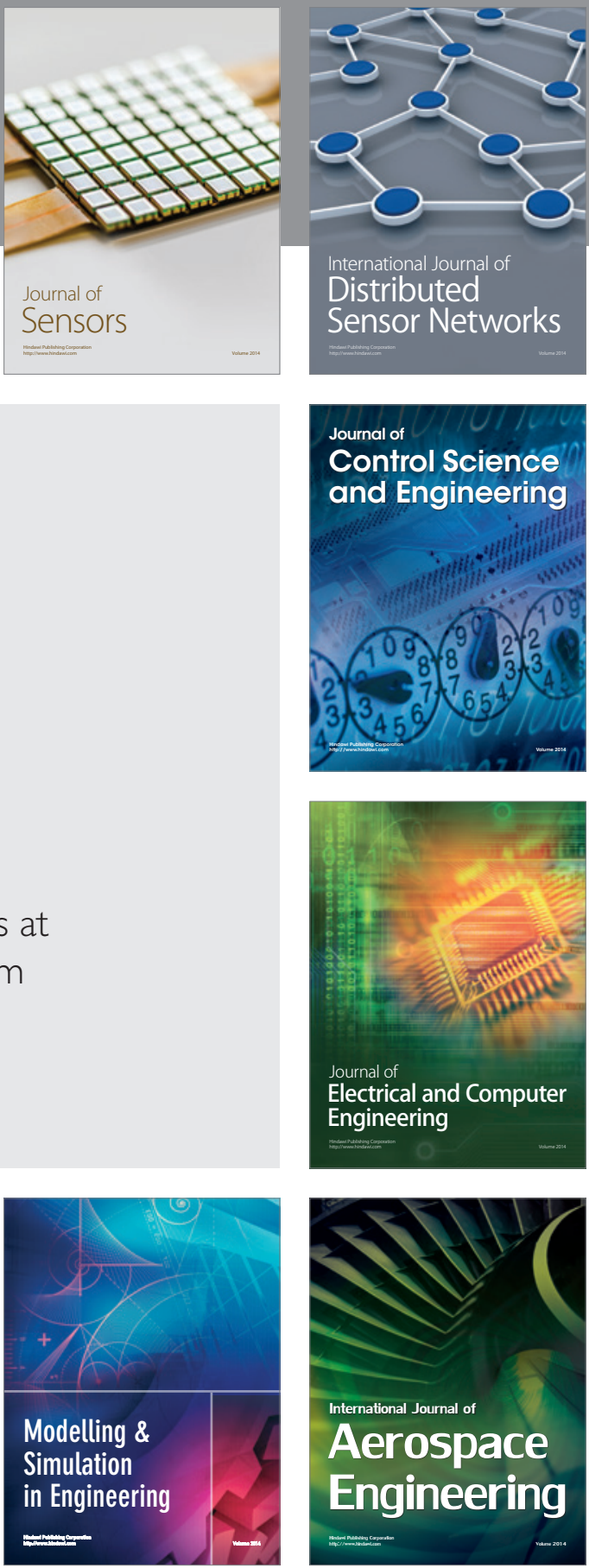

Journal of

Control Science

and Engineering
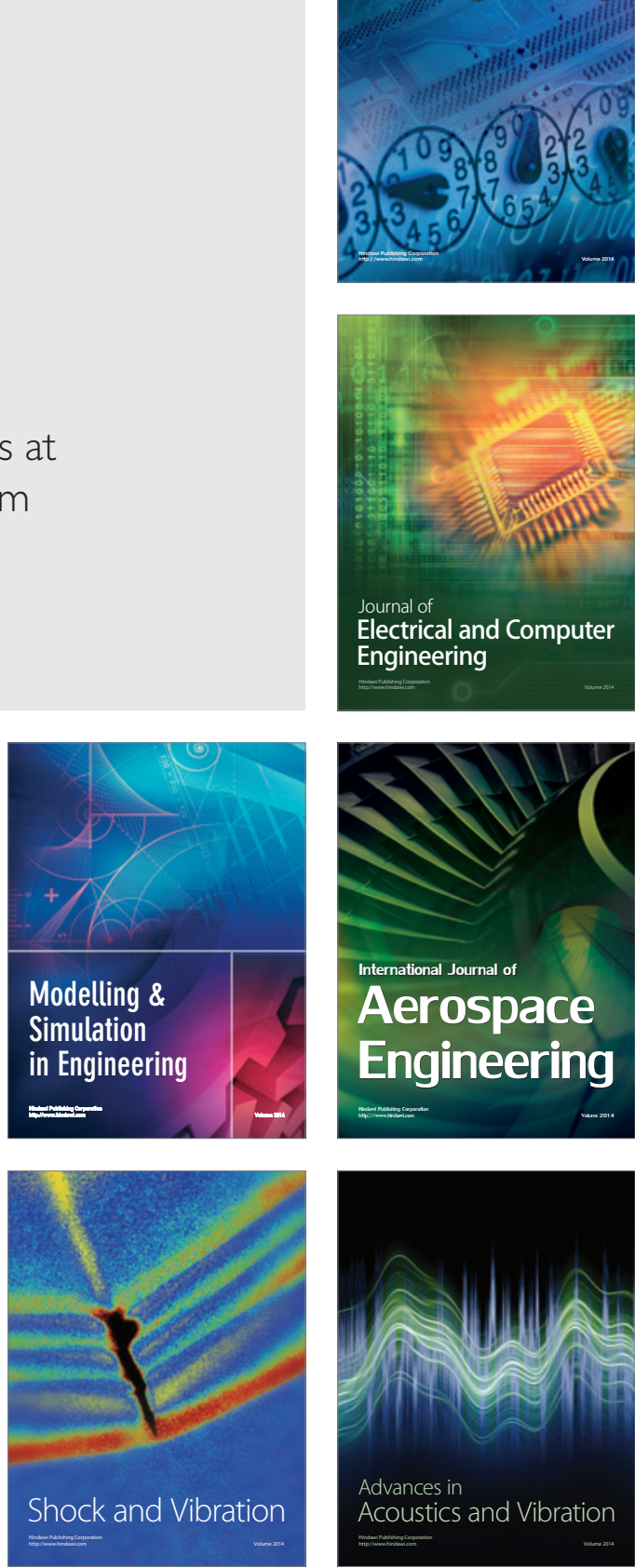